\title{
Interaction Energy Analysis of Monovalent Inorganic Anions in Bulk Water Versus Air/Water Interface
}

\author{
John M. Herbert*iD, Suranjan K. Paul \\ 1 Department of Chemistry \& Biochemistry, The Ohio State University, Columbus, Ohio USA \\ * Correspondence: herbert@chemistry.ohio-state.edu
}

\begin{abstract}
Soft anions exhibit surface activity at the air/water interface that can be probed using surface-sensitive vibrational spectroscopy, yet the statistical mechanics behind this surface activity remains a matter of debate. Here, we examine the nature of anion-water interactions at the air/water interface using a combination of molecular dynamics simulations and quantum-mechanical energy decomposition analysis based on symmetry-adapted perturbation theory. Results are presented for a set of monovalent anions including $\mathrm{Cl}^{-}, \mathrm{Br}^{-}, \mathrm{I}^{-}, \mathrm{CN}^{-}, \mathrm{OCN}^{-}, \mathrm{SCN}^{-}, \mathrm{NO}_{2}^{-}, \mathrm{NO}_{3}^{-}$, and $\mathrm{ClO}_{n}^{-}$ $(n=1,2,3,4)$, several of which are archetypal examples of surface-active ions. In all cases, we find that anion-water interaction energies are systematically larger in bulk water although the difference (with respect to the interaction energy in the interfacial environment) is well within the magnitude of the instantaneous fluctuations. Specifically for the surface-active species $\mathrm{Br}^{-}(\mathrm{aq}), \mathrm{I}^{-}(\mathrm{aq}), \mathrm{ClO}_{4}^{-}(\mathrm{aq})$, and $\mathrm{SCN}^{-}(\mathrm{aq})$, and also for $\mathrm{ClO}^{-}(\mathrm{aq})$, the charge-transfer (CT) energy is found to be slightly larger at the interface than it is in bulk water, but in all cases the CT stabilization amounts to $<20 \%$ of the total induction energy. CT-free polarization energies are systematically larger in bulk water, for all of the ions. This analysis complements our recent work suggesting that the short-range solvation structure around these ions is scarcely different at the air/water interface from what it is in bulk water. Together, these observations suggest that changes in first-shell hydration structure around soft anions cannot explain observed surface activities.
\end{abstract}

Keywords: air-water interface, Hofmeister series; hydrogen bonding; charge transfer; symmetryadapted perturbation theory; noncovalent interactions

\section{Introduction}

One of the earliest results of surface-sensitive vibrational sum-frequency generation (VSFG) experiments [1] was the observation that soft anions impact the O-H vibrational lineshape but that hard anions do not [2-5]. The term "soft" is chosen carefully here, as an alternative to "polarizable", and can be roughly interpreted as "monovalent and polarizable", equivalent to having a low surface charge density [6]. (Such ions are sometimes called "chaotropic" [7].) Although the surface activity of certain anions is often discussed in terms of polarizability [8-16], it should be noted that polyvalent anions such as $\mathrm{SO}_{4}^{2-}(\mathrm{aq})$ are quite polarizable [17], however the presence of such ions in solution does not affect the $\mathrm{O}-\mathrm{H}$ lineshape measure in VSFG experiments [18]. Molecular dynamics (MD) simulations suggest that hard anions, including polyvalent species but also fluoride, are repelled from the air/water interface $[19,20]$.

The nature of the surface activity in the soft anions remains a matter of debate. Whereas continuum electrostatics predicts that ions are repelled from the air/water interface [12], early MD simulations using polarizable force fields suggested that soft anions are not only present at the interface but in fact partition preferentially there $[8,12,19,21]$. More recent work, however, has suggested that these concentration enhancements were exaggerated by the force fields in use at the time [22-26], which aligns with the interpretation of some of the early experiments [2]. According to this point of view, surface activity may simply reflect the absence of depletion of soft anions at the interface $[27,28]$, rather than a concentration enhancement.

To this debate, the present authors have recently added the observation (based upon MD simulations using polarizable force fields) that the first-shell hydration structure 
around soft anions is hardly different at the air/water interface as compared to values computed for the same anions in bulk water [6]. Such similarities had been noted previously for $\mathrm{I}^{-}$(aq) [29] and for $\mathrm{SCN}^{-}$(aq) [30], and this is notable since iodide and thiocyanate are archetypal examples of ions that perturb the $\mathrm{O}-\mathrm{H}$ lineshape in VSFG experiments [2,3, 18,31,32]. Our work considered a much larger set of anions [6], and the close structural similarities that we observe (including both the average number as well as the orientation of the ion-water hydrogen bonds) suggest that the origins of anion-induced changes in the $\mathrm{O}-\mathrm{H}$ vibrational lineshape must be rather subtle effects on water-water hydrogen bonds, perhaps due to ion-induced changes in local electric fields.

Our previous work [6] was limited to structural characterization of the ions in question, along with a detailed examination of their ionization energies in order to make contact with liquid microjet photoelectron spectroscopy [33]. The present work adds an energetic component to this analysis. Specifically, we compute anion-water interaction energies for the same set of monovalent anions that we considered previously: $\mathrm{Cl}^{-}, \mathrm{Br}^{-}, \mathrm{I}^{-}, \mathrm{CN}^{-}$, $\mathrm{OCN}^{-}, \mathrm{SCN}^{-}, \mathrm{NO}_{2}^{-}, \mathrm{NO}_{3}^{-}$, and $\mathrm{ClO}_{n}^{-}(n=1,2,3,4)$. Some of these are typical surfaceactive ions (e.g., $\mathrm{Br}^{-}, \mathrm{I}^{-}, \mathrm{SCN}^{-}$, and $\mathrm{ClO}_{4}^{-}$), whereas others such as $\mathrm{CN}^{-}, \mathrm{OCN}^{-}$, and $\mathrm{NO}_{2}^{-}$visit the interface much less frequently, according to the MD simulations [6], and are not classified as surface-active. (They do still spend enough time near the interface that it is possible to construct an interfacial data set, even in these cases, which then presents a useful comparison to the canonical surface-active anions.) Intermediate cases with weak surface activity include $\mathrm{Cl}^{-}$and $\mathrm{NO}_{3}^{-}$[18]. We present a detailed analysis of the (ensembleaveraged) interaction between each of these ions and its short-range hydration sphere, in both bulk water and at the air/water interface, using the quantum-chemical methods of symmetry-adapted perturbation theory (SAPT) [34-37]. The SAPT family of methods [34, 35] is designed both for accurate calculation of noncovalent interaction energies as well as a physically-motivated energy decomposition analysis of those energies $[35,36]$. Of key interest will be whether the interfacial environment engenders any discernible changes in the ion-water interactions, relative to what is observed for the same ion in bulk water.

\section{Computational Methods}

\subsection{Classical MD Simulations}

MD simulations of the aforementioned ions, in a periodic slab configuration, were reported in Ref. 6 and the same set of simulations is used here to obtain snapshots for interaction energy analysis. These simulations were performed under NVT conditions at $T=298 \mathrm{~K}$ and a bulk density of $0.997 \mathrm{~g} / \mathrm{cm}^{3}$, and the size of the periodic simulation cell (31.3 $\AA \times 31.3 \AA \times 156.7 \AA)$ was previously shown to afford converged results [6]. The simulation data were subsequently partitioned into bulk and interfacial parts depending on the position of the ion relative to the Gibbs dividing surface (GDS) that we take to define the air/water interface. For the snapshots classified as "interfacial", the ion's center of mass lies no more than $3 \AA$ below the GDS. Anything beyond this cutoff is considered to be a bulk water environment, as this interior region of the periodic slab affords properties that are essentially indistinguishable from results performed in an isotropic simulation that has no interface [6]. Simulations were performed using the AMOEBA force field for water [38], whose parameterization includes some of the ions in question, such as the halides [39]. Parameters for the remaining ions were developed in Ref. 6 along similar lines, following an established protocol [40], and are included in the Supplementary Material. Energetic analyses with the AMOEBA force field were performed using the Tinker software, v. 8 [41].

Following an equilibration period, snapshots were extracted that include two solvation shells around the ion, according to the distance criteria described in Ref. 6. The number of water molecules varies from one snapshot to the next, with the average number $\left\langle N_{\mathrm{w}}\right\rangle$ depending on both the size of the ion and how tightly hydrated it is. In bulk water, these averages range from $\left\langle N_{\mathrm{w}}\right\rangle \approx 28$ for $\mathrm{Cl}^{-}(\mathrm{aq})$ up to $\left\langle N_{\mathrm{w}}\right\rangle \approx 43-44$ for $\mathrm{Br}^{-}(\mathrm{aq})$ and $\mathrm{I}^{-}(\mathrm{aq})$, with $\left\langle N_{\mathrm{w}}\right\rangle=35-37$ for the remaining ions. The interfacial snapshots contain fewer water molecules, on average, as the water density is smaller in the interfacial region. In the 
analysis that follows, we consider interaction energies $\left(E_{\text {int }}\right)$ between the ion and its first two hydration shells. The quantity $E_{\text {int }}$ is intensive with respect to system size and this insulates our analysis against the step-to-step fluctuations in the number of water molecules that are included in these calculations. Ensemble averages reported below represent 51 snapshots for each ion in bulk water as well as 51 snapshots for each ion at the air/water interface, with each individual snapshot separated by 5 ps in time in the corresponding MD simulation. Coordinate files for these data sets are provided in the Supplementary Material.

\subsection{Symmetry-Adapted Perturbation Theory}

Quantum-mechanical values of $E_{\text {int }}$ were computed using SAPT based on HartreeFock (HF) wave functions for the monomers and second-order perturbation theory for the intermolecular Coulomb operators, a method that is usually called SAPT0 [35,42,43], and which is closely related to second-order Møller-Plesset perturbation theory (MP2). However, because second-order dispersion is far from quantitative $[34,35,44]$, we replace it in these calculations with a many-body dispersion (MBD) model $[35,45,46]$, in what we have termed a "hybrid" or "extended" form of SAPT [35]. This method will be designated as SAPT0 + MBD. At this level of theory, results for small-molecule data sets suggests that errors in $E_{\text {int }}$ are within $\sim 1 \mathrm{kcal} / \mathrm{mol}$ of the best-available benchmarks [43,46], provided that adequate basis sets are employed [43]. All electronic structure calculations were performed using the Q-Chem software, v. 5 [47]. 35]

The interaction energy computed using SAPT0 + MBD is naturally partitioned as [34,

$$
E_{\text {int }}=E_{\text {elst }}+E_{\text {exch }}+E_{\text {ind }}+E_{\text {disp }} .
$$

The terms on the right represent electrostatics $\left(E_{\text {elst }}\right)$, meaning the Coulomb interaction between isolated-monomer charge densities; exchange or Pauli repulsion $\left(E_{\text {exch }}\right)$, which is the cost to antisymmetrize the isolated-monomer wave functions; induction $\left(E_{\text {ind }}\right)$; and dispersion $\left(E_{\text {disp }}\right)[35,48]$. In our approach,

$$
E_{\text {elst }} \equiv E_{\text {elst }}^{(1)}
$$

and

$$
E_{\text {exch }} \equiv E_{\text {exch }}^{(1)}
$$

in Eq. (1) are the first-order SAPT electrostatic and exchange energies, while $E_{\text {disp }}$ is the dispersion energy computed using the MBD model [46]. The induction energy comes from second-order SAPT but warrants additional discussion and is detailed in Section 2.3.

Previous basis-set testing of SAPT0 + MBD reveals that polarized triple- $\zeta$ basis sets are both necessary and sufficient to obtain converged energetics [34,43]. This is a unique feature of our hybrid approach to SAPT [35], which replaces the very slow basis-set convergence of perturbative dispersion with a model (MBD) that converges quickly, with the density. Tests for $\mathrm{Cl}^{-}(\mathrm{aq})$ in Fig. 1 demonstrate that interaction energies computed using the 6$311+\mathrm{G}(\mathrm{d}, \mathrm{p})$ basis set agree with SAPT0 + MBD/def2-TZVPD values to within an average of $2.0 \mathrm{kcal} / \mathrm{mol}$, in a total interaction energy that averages $-106 \mathrm{kcal} / \mathrm{mol}$. Relative to the more complete def2-TZVPD basis set, the Pople basis set systematically underestimates $E_{\text {ind }}$ (by an average of $1.6 \mathrm{kcal} / \mathrm{mol}$ ) and overestimates $E_{\text {elst }}$ (by an average of $4.2 \mathrm{kcal} / \mathrm{mol}$ ), whereas $E_{\text {exch }}$ and $E_{\text {disp }}$ are nearly identical in both basis sets.

More important than these relatively small differences is the fact that instantaneous values of $E_{\text {int }}$ fluctuate from snapshot to snapshot in a similar way in either basis set. For these calculations, which involve $\mathrm{Cl}^{-}\left(\mathrm{H}_{2} \mathrm{O}\right)_{n}$ with an average of $n=28$ water molecules, $\mathrm{SAPT} 0+\mathrm{MBD} / 6-311+\mathrm{G}(\mathrm{d}, \mathrm{p})$ calculations are $17 \times$ faster than the corresponding calculations with def2-TZVPD. (This speedup results largely from the absence of diffuse functions on hydrogen but also benefits from Q-Chem's very efficient handling of $s p$ shells in Pople 


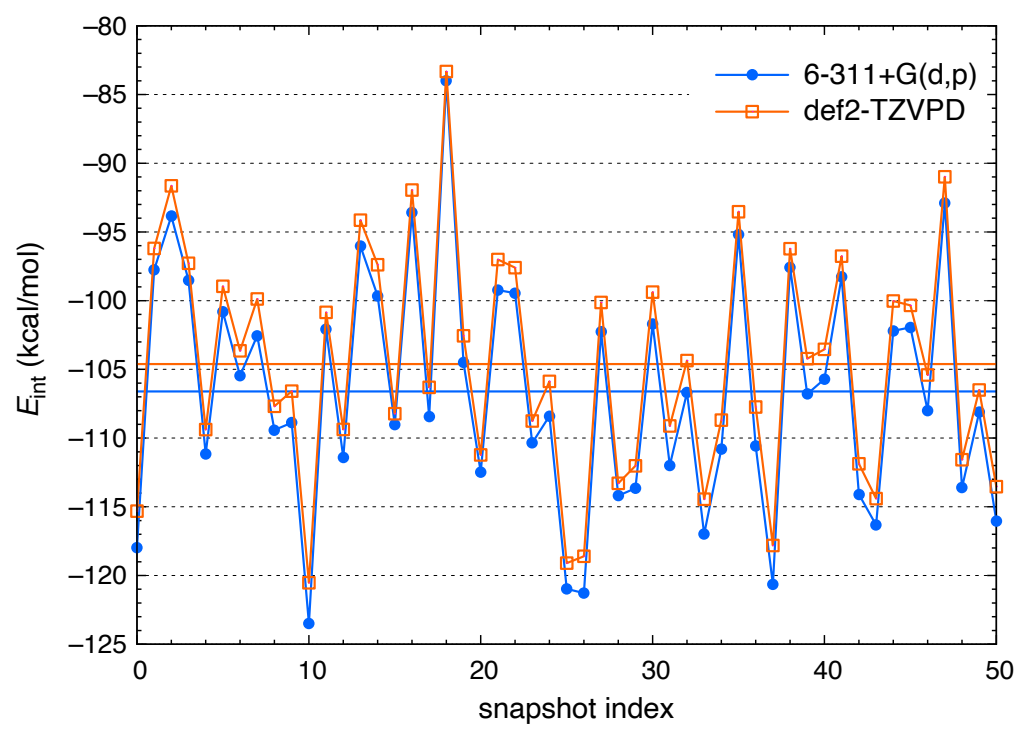

Figure 1. Total interaction energies for snapshots of $\mathrm{Cl}^{-}(\mathrm{aq})$ in bulk water, computed at the SAPT0 + MBD level using two different basis sets. Solid horizontal lines show the ensemble-averaged values obtained using either basis set. These averages are $\left\langle E_{\text {int }}\right\rangle=-106.6 \pm 8.4 \mathrm{kcal} / \mathrm{mol}$ for 6$311+\mathrm{G}(\mathrm{d}, \mathrm{p})$ and $\left\langle E_{\text {int }}\right\rangle=-104.6 \pm 8.2 \mathrm{kcal} / \mathrm{mol}$ for def2-TZVPD, where the uncertainties represent one standard deviation.

basis sets.) In the present work, we are concerned with comparisons between bulk and interfacial behavior rather than absolute interaction energies, and the need for ensemble averaging requires high throughput. As such, $6-311+G(d, p)$ is used for all subsequent SAPT calculations.

Interaction energies defined in Eq. (1) do not include relaxation of the monomer geometries, so $E_{\text {int }}$ is an interaction energy in the "vertical" sense, not a binding energy or a solvation energy. In considering the ion-water clusters $\mathrm{X}^{-}\left(\mathrm{H}_{2} \mathrm{O}\right)_{n}$ extracted from MD simulations, we treat the entire water cluster $\left(\mathrm{H}_{2} \mathrm{O}\right)_{n}$ as a single monomer for the purpose of computing $E_{\text {int }}$ and its components, then average over the ensemble of snapshots. Even so, the value $\left\langle E_{\text {int }}\right\rangle$ corresponds to vertical removal of the ion. It includes the change in (electronic) polarization of the water molecules upon removal of the ion but does not include the (orientational) reorganization energy of the water to fill the void left behind by the ion.

Unless otherwise specified, all of the SAPT0 calculations reported herein use HF wave functions for the monomers. However, we will report a few SAPTO(KS) calculations $[35,43]$ in which Kohn-Sham (KS) molecular orbitals from density functional theory (DFT) are used in place of HF orbitals. These SAPTO(KS) calculations employ the long-range corrected (LRC) density functional LRC- $\omega$ PBE [49]. Previous work has emphasized the importance of using an asymptotically correct exchange potential in SAPT calculations $[43,44,50,51]$, and this condition can be achieved in practice via monomer-specific tuning of the rangeseparation parameter $(\omega)$ in LRC- $\omega$ PBE functional. Although "optimal tuning" of LRC functionals $[52,53]$ is sometimes accomplished using the ionization energy (IE) theorem of DFT,

$$
\mathrm{IE}=-\varepsilon_{\mathrm{HOMO}},
$$

a more robust procedure in the present context is the "global density-dependent" (GDD) or " $\omega_{\mathrm{GDD}}$ " procedure $[43,44,51]$. This approach, which adjusts $\omega$ based on the size of the exchange hole, mitigates the strong dependence on system size that is observed when using IE tuning [43], and which might otherwise be a problem when studying water clusters of varying size [54]. For water, we use $\omega=0.277 a_{0}^{-1}$, which represents an average over several cluster geometries. For the ions, we tune $\omega$ individually at the optimized 
gas-phase geometry of each, resulting in a range of values from $\omega=0.248 a_{0}^{-1}$ for iodide and $\omega=0.261 a_{0}^{-1}$ for bromide, where the tails of the anion's density are most diffuse, up to $\omega=0.398 a_{0}^{-1}$ for cyanate and $\omega=0.405 a_{0}^{-1}$ for cyanide, where the density is most compact. (Recall that LRC functionals switch from semilocal to HF exchange on a length scale of $\sim 1 / \omega$.)

In previous work we have often used self-consistent charge embedding of the SCF monomer wave functions as a means to incorporate many-body polarization effects into a pairwise SAPT calculation, albeit implicitly [34,55-58]. The present work does not make use of any charge embedding, however, and instead the $\mathrm{X}^{-}\left(\mathrm{H}_{2} \mathrm{O}\right)_{n}$ system is treated as dimers, with $\left(\mathrm{H}_{2} \mathrm{O}\right)_{n}$ as one monomer. In principle, charge embedding could be used to describe these clusters more efficiently as $(n+1)$-body systems with monomers $\mathbf{X}^{-}$ and $\mathrm{H}_{2} \mathrm{O}$, but we have chosen not to do so here. The dimer approach makes the SAPT interaction energies more directly comparable to those obtained using the AMOEBA force field.

\subsection{Polarization and Charge Transfer}

In our calculations, the induction term in Eq. (1) is defined as

$$
E_{\text {ind }}=E_{\text {ind }}^{(2)}+E_{\text {exch-ind }}^{(2)}+\delta E_{\mathrm{HF}},
$$

where the first two terms are the second-order (SAPT0) induction and exchange-induction energies, and

$$
\delta E_{\mathrm{HF}}=\Delta E_{\mathrm{int}}^{\mathrm{HF}}-\left(E_{\text {elst }}^{(1)}+E_{\text {exch }}^{(1)}+E_{\text {ind,resp }}^{(2)}+E_{\text {exch-ind,resp }}^{(2)}\right)
$$

is the so-called " $\delta \mathrm{HF}^{\prime}$ correction [35]. It uses a counterpoise-corrected, supramolecular $\mathrm{HF}$ interaction energy $\left(\Delta E_{\mathrm{int}}^{\mathrm{HF}}\right)$ to correct the SAPT0 interaction energy for induction effects beyond second order in perturbation theory, which is crucial for the accurate description of hydrogen bonds [35,43]. See Ref. 59 for a definition of the second-order response ("resp") energies that appear in Eq. (5).

As defined in SAPT, the induction energy in Eq. (4) contains both polarization and charge transfer $(\mathrm{CT})$,

$$
E_{\text {ind }}=E_{\text {pol }}+E_{\mathrm{CT}},
$$

for reasons that are discussed in Ref. [60]. In the analysis of hydrogen bonding it is often of interest to separate these effects but that separation has historically been considered problematic, and not just within the SAPT formalism; many schemes for separating polarization from CT exhibit strong dependence on the choice of basis set [60]. To accomplish the separation in Eq. (6) in a robust way that converges rapidly with respect to basis set, we use the machinery of a charge-constrained self-consistent field (SCF) calculation [61] in order to define a CT-free reference state. Here, the monomers are allowed to polarize one another but their charge densities are constrained to integrate to integer numbers of electrons. Because the SCF procedure is variational, lifting of this constraint necessarily lowers the energy (to that of the fully-relaxed SCF solution), and this energy lowering is taken to define $E_{\mathrm{CT}}$. The CT energy thus obtained is then subtracted from the induction energy to obtain the CT-free polarization energy, $E_{\text {pol }}=E_{\text {ind }}-E_{\mathrm{CT}}[60,62-64]$. CT energies defined in this way are very nearly converged already in double- $\zeta$ basis sets [60]. This approach has previously been used to demonstrate that $E_{\mathrm{CT}}$ furnishes a driving force for formation of quasi-linear hydrogen bonds in binary halide-water complexes [48,64].

Implementation of the charge-constrained SCF procedure requires a method to count electrons, and Becke's multicenter partition scheme [65] is commonly used for this purpose [61]. This approach divides space into Voronoi cells [66], which are regions of space that are closest to a particular nucleus, and then Becke applies a smoothing function at the boundaries of these polyhedra. Alternatively, and specifically for the purpose of defining a CT-free reference state in order to effect the partition suggested in Eq. (6), a counting proce- 


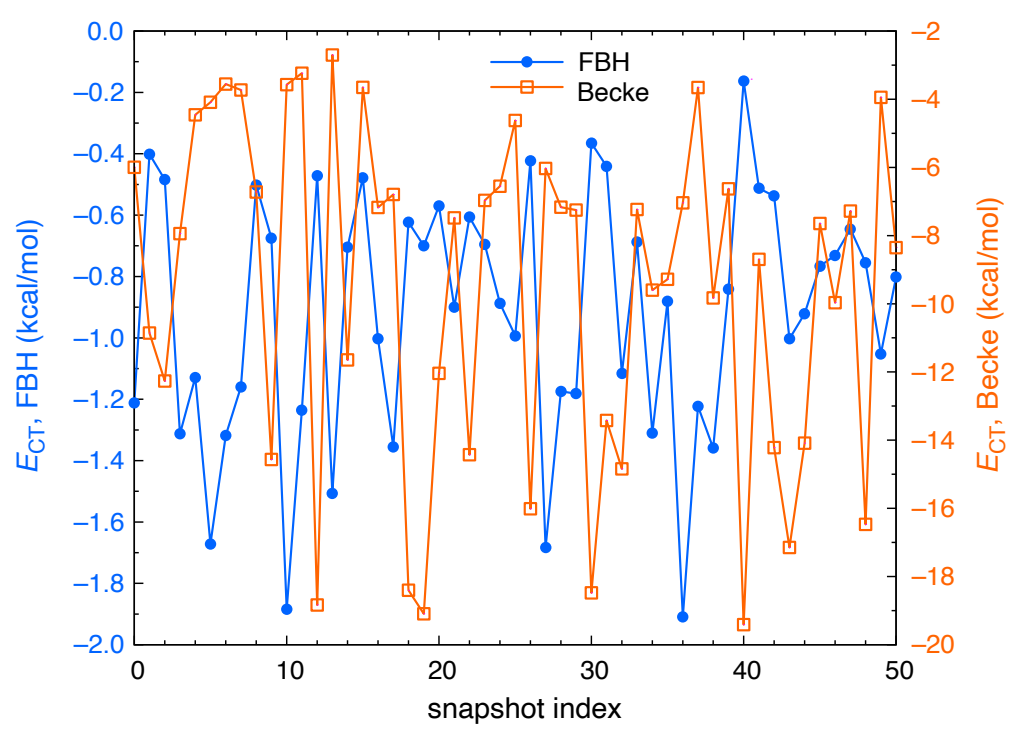

Figure 2. CT energies for snapshots of $\mathrm{I}^{-}(\mathrm{aq})$ in bulk water, computed using a charge-constrained SCF procedure with the charge constraint defined either using fragment-based Hirshfeld (FBH) weights (scale at left), or else Becke's multicenter partitioning procedure (scale at right). Results using the Becke scheme include the "atomic size adjustments" that are described in Ref. 65, wherein Slater's set of atomic radii [67] are used to adjust the boundaries of the Voronoi cells based on atomic size.

dure based on fragment-based Hirshfeld (FBH) weighting has also been suggested [62,64]. In the latter approach, the number of electrons contained in fragment $A$ is defined as

$$
N_{A}=\int w_{A}(\mathbf{r}) \rho(\mathbf{r}) d \mathbf{r},
$$

where $\rho(\mathbf{r})$ is the supramolecular electron density, which is integrated subject to a weighting function $w_{A}(\mathbf{r})$. That function is defined as

$$
w_{A}(\mathbf{r})=\frac{\rho_{A}^{0}(\mathbf{r})}{\sum_{B} \rho_{B}^{0}(\mathbf{r})}
$$

where $\rho_{X}^{0}(\mathbf{r})$ is the charge density of isolated fragment $X$. The denominator in Eq. (8) is thus a superposition of isolated-fragment densities.

The Becke scheme can also be conceptualized as a form of Eq. (7) in which $w_{A}(\mathbf{r})$ is a smoothed version of a Heaviside step function, which switches rapidly between $w_{A}(\mathbf{r})=0$ and $w_{A}(\mathbf{r})=1$ at the boundaries of the Voronoi polyhedra. In practice, our implementation of Becke's procedure uses the "atomic size adjustments" that are described in Ref. 65, in which a set of empirical atomic radii [67] are used to adjust the boundaries of the Voronoi cells away from the midpoints of the internuclear vectors. As discussed below, this adjustment is crucial for systems with substantial size mismatch between nearby atoms.

Even so, the FBH approach strikes us as the more reasonable one, especially where anions are involved, because Becke's approach depends only on the positions of the atoms (along with the empirical atomic radii), whereas the weight function defined in Eq. (8) respects the diffuseness of the isolated anions's wave function. In the present context, this almost inevitably means that the extent of anion $\rightarrow$ water $\mathrm{CT}$ is smaller when the FBH approach is used, because the tails of the $\mathrm{X}^{-}$wave function cause a larger region of space to contribute to that fragment's integrated electron number, $N_{X}$. As an example, Fig. 2 presents $E_{\mathrm{CT}}$ computed using both methods, for each snapshot of $\mathrm{I}^{-}(\mathrm{aq})$ in bulk water. The results are considerably different depending on which method is used to 


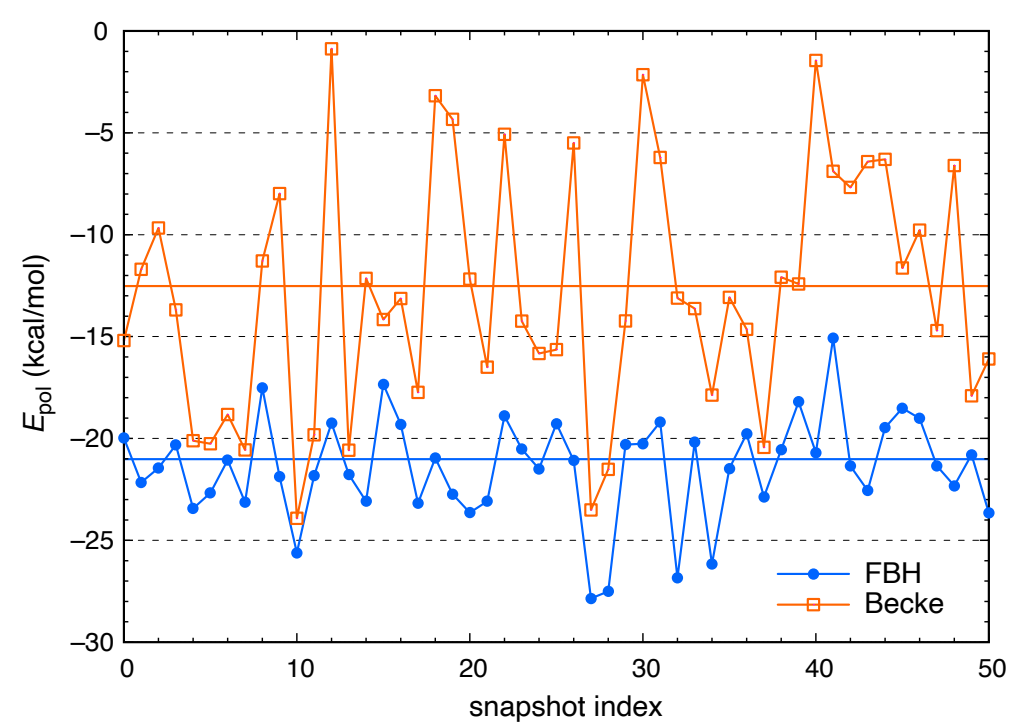

Figure 3. CT-free polarization energy $\left(E_{\mathrm{pol}}=E_{\text {ind }}-E_{\mathrm{CT}}\right)$ for snapshots of $\mathrm{I}^{-}(\mathrm{aq})$ in bulk water. This quantity is obtained by removing $E_{\mathrm{CT}}$ from the total SAPT0 induction energy using either of two schemes (FBH weighting or Becke partition with atomic size adjustments) to integrate the charge constraint that defines the CT-free reference state. Solid horizontal lines show the ensemble-averaged values, which are $\left\langle E_{\mathrm{pol}}\right\rangle=-21.0 \pm 3.9 \mathrm{kcal} / \mathrm{mol}(\mathrm{FBH})$ and $\left\langle E_{\mathrm{pol}}\right\rangle=-12.6 \pm 6.1 \mathrm{kcal} / \mathrm{mol}$ (Becke), where the uncertainties represent one standard deviation.

count electrons, with the FBH approach compressing the CT energy into the interval $0>E_{\mathrm{CT}}>-2 \mathrm{kcal} / \mathrm{mol}$ whereas the Becke procedure affords values of $\left|E_{\mathrm{CT}}\right|$ as large as $20 \mathrm{kcal} / \mathrm{mol}$. The latter value is comparable to the the average magnitude of the total SAPT0 induction energy, which is $\left\langle E_{\text {ind }}\right\rangle=-22.3 \mathrm{kcal} / \mathrm{mol}$ for $\mathrm{I}^{-}(\mathrm{aq})$ in bulk water. (Note that energy components corresponding to attractive interactions are negative.)

Figure 3 shows the polarization energy $\left(E_{\mathrm{pol}}=E_{\text {ind }}-E_{\mathrm{CT}}\right)$ that is obtained using either the Becke or the FBH weighting function to define the charge constraint. (Both definitions of $E_{\mathrm{pol}}$ start from the same SAPT0 induction energy, $E_{\mathrm{ind}}$.) It is apparent that the two definitions afford step-to-step fluctuations that do not seem to correlate with one another. In the Becke definition, the size and shape of the Voronoi cell that contains the iodide anion is sensitive to the instantaneous values of all iodide-water distances in the first solvation shell, whereas the FBH definition uses a spherically-symmetric charge density for the isolated anion in order to define the charge constraint; the latter definition is less sensitive to fluctuations in the atomic coordinates. (Note that FBH definition certainly remains sensitive to the presence of hydrogen bonds $[48,64]$.)

For $\mathrm{I}^{-}(\mathrm{aq})$, it is consistently the case that the CT-free reference state defined using Becke partition results in CT energies that are larger in magnitude: $\mid E_{\mathrm{CT}}($ Becke $)|>| E_{\mathrm{CT}}(\mathrm{FBH}) \mid$. This is evident from the rather different energy scales in Fig. 2, but the situation is not the same for all of the anions. As a second example we consider $\mathrm{ClO}^{-}(\mathrm{aq})$, which exhibits the largest values of $\left|E_{\mathrm{CT}}\right|$ of any of the ions studied here, at least when the $\mathrm{FBH}$ definition is used. Figure 4 considers both definitions and examines how $E_{\mathrm{CT}}$ fluctuates from snapshot to snapshot. Becke's partition predicts very little $\mathrm{CT}$ for $\mathrm{ClO}^{-}$in bulk water $\left(\left\langle E_{\mathrm{CT}}\right\rangle=-1.2 \mathrm{kcal} / \mathrm{mol}\right)$ whereas the $\mathrm{FBH}$ definition results in an average value of $\left\langle E_{\mathrm{CT}}\right\rangle=-6.2 \mathrm{kcal} / \mathrm{mol}$. In either case, $E_{\mathrm{CT}}$ is consistently larger for the interfacial snapshots.

We will use the FBH-based definition for the remainder of this work, and our main interest is in understanding how various energy components compare when the ion is in a bulk versus an interfacial environment. As noted in the examples presented above, the magnitude of $E_{\mathrm{CT}}$ can depend strongly on the method that is used to count electrons. This observation suggests that in other applications of constrained DFT [61], which is the 


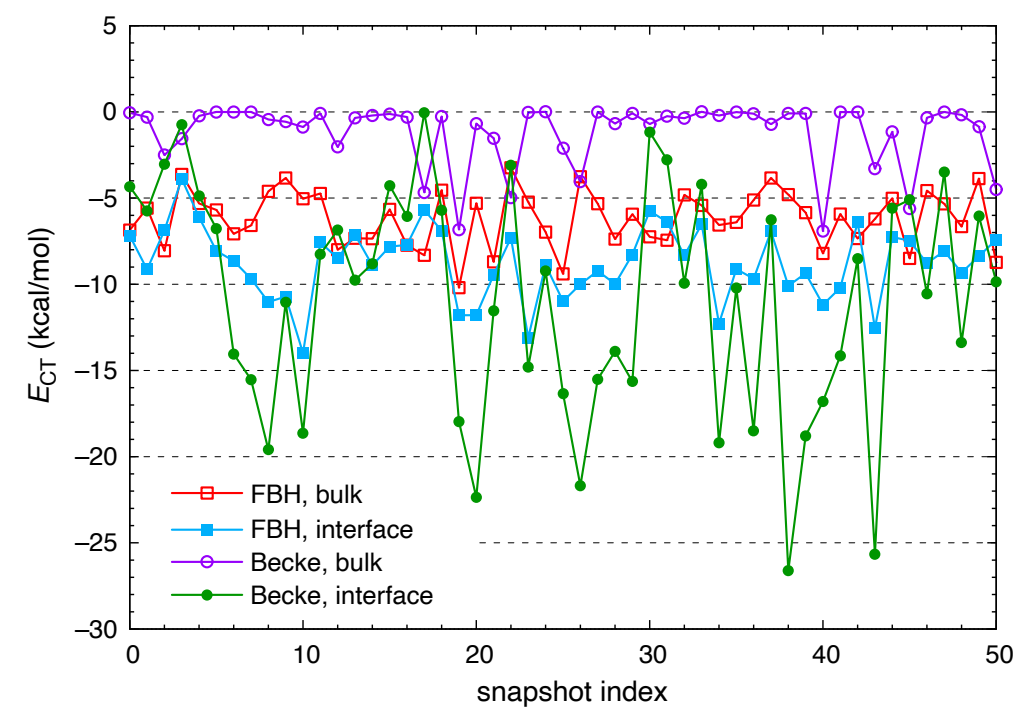

Figure 4. $\mathrm{CT}$ energies for $\mathrm{ClO}^{-}(\mathrm{aq})$, computed using either Becke partition or else $\mathrm{FBH}$ weighting to define the CT-free reference state, and considering both the bulk and interfacial data sets.

more common form of charge-constrained SCF calculation (in contrast to the constrained HF calculations employed here), the results should be checked carefully to ensure that conclusions are robust with respect to the details of how the constraints are implemented.

The SG-3 quadrature grid [68] is used to integrate the SCF constraint equations as well as Eq. (7). As a technical aside, we note that the atomic size adjustments mentioned above are crucial in order to obtain results that are even remotely sensible, when Becke partition is used to implement the charge constraint. However, the original implementation of the charge-constrained SCF procedure in the Q-Chem program did not include these corrections [69], for reasons that are unclear because the same algorithm with these size adjustments was implemented in the NWChem program, by the same authors at around the same time [70]. Atomic size corrections were later added to Q-Chem's version of Becke partition for the purpose of SAPT-based CT analysis [64]. Absent these corrections, the Voronoi cell boundaries are placed at midpoints of the internuclear vectors, which affords unreasonable results in cases where neighboring atoms have very different size. This includes covalent bonds to hydrogen, where the midpoint definition causes too much density to be assigned to the smaller hydrogen atom, often leading to a negative charge assigned to hydrogen [64]. In the present work, neglecting the atomic size corrections leads to a significant fraction of the iodide's charge being assigned to first-shell water molecules, resulting in completely unrealistic CT energies whose magnitudes exceed the total SAPT0 induction energy. In our view, constrained DFT based on Becke partition should probably never be used without the atomic size corrections.

\section{Results and Discussion}

Figure 5 presents ensemble-averaged interaction energies for the sets of $\mathrm{X}^{-}\left(\mathrm{H}_{2} \mathrm{O}\right)_{n}$ structures that are considered here, where $\mathrm{X}^{-}$is one of 12 monovalent inorganic anions. Two solvation shells of surrounding water are treated as a single monomer for the purpose of the SAPT calculations. Results are presented both at the quantum-mechanical SAPT0 + MBD $/ 6-311+G(d, p)$ level [Fig. 5(a)] and also using the AMOEBA force field [Fig. $5(\mathrm{~b})]$, where the latter is the same force field that was used for the simulations from which these $\mathrm{X}^{-}\left(\mathrm{H}_{2} \mathrm{O}\right)_{n}$ structures were extracted. Bulk and interfacial data are averaged separately, with the criterion GDS $-3 \AA$ used to decide whether a particular snapshot represents a bulk or an interfacial solvation environment. 

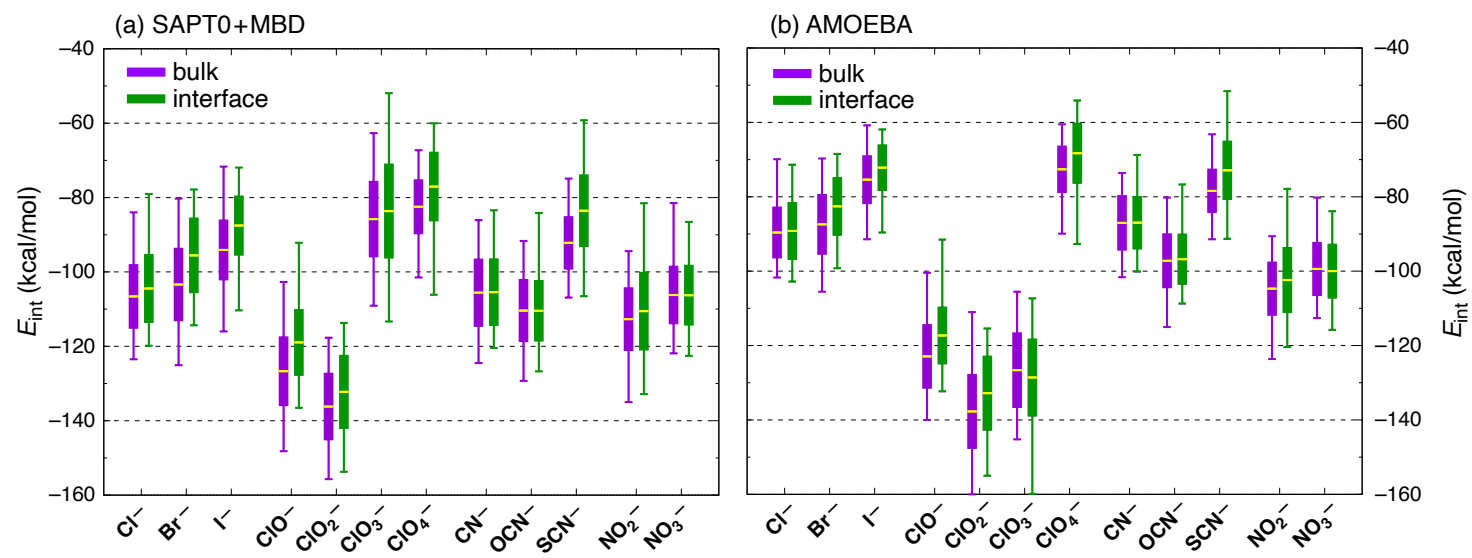

Figure 5. Total ion-water interaction energies between various anions and their first two hydration shells, computed using (a) quantum chemistry at the level of SAPT0 + MBD/6-311+G(d,p), versus (b) the AMOEBA force field that was used to obtain the structures. Side-by-side box and whisker plots are shown for each ion in bulk and interfacial environments. Box plots show the mean value $\left\langle E_{\text {int }}\right\rangle$ (yellow bar) and extend for one standard deviation in both directions. Whiskers indicate minimum and maximum values of $E_{\text {int }}$.

There are two interesting observations to be made from the interaction energy data in Fig. 5. Foremost is the fact that differences between the bulk and interfacial mean values $\left\langle E_{\text {int }}\right\rangle$ for a given ion are small compared to the fluctuations in the instantaneous value of $E_{\text {int }}$. Bulk values of $\left\langle E_{\text {int }}\right\rangle$ are systematically (slightly) larger in magnitude than interfacial values, except for $\mathrm{CN}^{-}, \mathrm{OCN}^{-}$, and $\mathrm{NO}_{3}^{-}$where the averages are essentially identical. In all cases, however, the difference between bulk and interfacial average values of $E_{\text {int }}$ is well within the standard deviation in either quantity; see the numerical values that are provided in Table 1. For the halides, the modest reductions in $\left\langle E_{\text {int }}\right\rangle$ at the interface (up to $7-8 \mathrm{kcal} / \mathrm{mol}$ for bromide and iodide) are consistent with results from classical MD simulations indicating that the average ion-water interaction is reduced, for all of the halides, as the ion moves towards the interface [20]. It should be noted that the simulations reported in Ref. 20 indicate that the enthalpic portion of the potential of mean force is more favorable for the heavier halides at the interface, as compared to its value in bulk water. As such, the rather subtle differences between ion-water interactions that are documented in our quantum-mechanical calculations are more than compensated by ion-induced changes in the water-water interactions [20]. This is consistent with our detailed structural analysis of the ions [6], which indicates very little change in the first-shell structure at the interface as compared to that in bulk water.

A second interesting observation is the generally strong correlation between classical (AMOEBA) and quantum-mechanical (SAPT) values of $E_{\text {int }}$, even if the former are systematically smaller than the latter, e.g., by $15-19 \mathrm{kcal} / \mathrm{mol}$ for the halide ions. (These systematic differences are smaller for the other ions except in the case of $\mathrm{ClO}_{3}^{-}$, which is discussed below.) For the halide ions, we use AMOEBA parameters that were originally developed by Ponder and co-workers [39], and we note that the discrepancies between the force field and the quantum chemistry that are documented in Fig. 5 are much larger than those reported in Ref. 39 for binary $\mathrm{X}^{-}\left(\mathrm{H}_{2} \mathrm{O}\right)$ complexes. This underscores the importance of considering larger ion-water clusters, given the many-body nature of polarization in aqueous systems [71-76]. Simulation of the hydration free energy of $\mathrm{Cl}^{-}$using AMOEBA results in an error of $11.9 \mathrm{kcal} / \mathrm{mol}$ with respect to experiment [39], assuming that the reference value is defined using the proton solvation energy of Tissandier et al. [77], which has since emerged as the consensus value [78-80]. In view of this, the systematic difference of $17 \mathrm{kcal} / \mathrm{mol}$ between AMOEBA and SAPT0 + MBD values of $\left\langle E_{\text {int }}\right\rangle$ in bulk water (see Table 1) is not so dissimilar from previous results. Improvements to the AMOEBA force field for ions, using SAPT energy components as benchmark data, is a topic of contemporary interest [81-83]. 
Table 1. Ensemble-averaged interaction energies $\left(E_{\mathrm{int}}\right)$ and induction energies $\left(E_{\mathrm{ind}}\right)$ computed at the SAPT0 $+\mathrm{MBD} / 6-311+\mathrm{G}(\mathrm{d}, \mathrm{p})$ level, including the $\delta$ HF correction for induction. ${ }^{a}$

\begin{tabular}{lccccc}
\hline \multirow{2}{*}{ Ion } & \multicolumn{2}{c}{$\left\langle E_{\text {int }}\right\rangle(\mathbf{k c a l} / \mathbf{m o l})^{b}$} & & \multicolumn{2}{c}{$\left\langle E_{\text {ind }}\right\rangle(\mathbf{k c a l} / \mathbf{m o l})^{c}$} \\
\cline { 2 - 3 } \cline { 5 - 6 } & bulk & interface & & bulk & interface \\
\hline $\mathrm{Cl}^{-}$ & $-106.6 \pm 8.4$ & $-104.4 \pm 9.0$ & & $-26.4 \pm 2.4$ & $-24.6 \pm 2.7$ \\
$\mathrm{Br}^{-}$ & $-103.4 \pm 9.6$ & $-95.5 \pm 9.9$ & & $-24.6 \pm 2.7$ & $-22.4 \pm 3.3$ \\
$\mathrm{I}^{-}$ & $-94.1 \pm 7.9$ & $-87.5 \pm 7.8$ & & $-22.3 \pm 2.7$ & $-20.3 \pm 2.8$ \\
$\mathrm{ClO}^{-}$ & $-126.7 \pm 9.1$ & $-118.9 \pm 8.7$ & & $-47.0 \pm 5.1$ & $-44.5 \pm 4.3$ \\
$\mathrm{ClO}_{2}^{-}$ & $-136.2 \pm 8.8$ & $-132.2 \pm 9.7$ & & $-52.3 \pm 4.7$ & $-50.2 \pm 5.3$ \\
$\mathrm{ClO}_{3}^{-}$ & $-85.8 \pm 10.0$ & $-83.6 \pm 12.5$ & & $-42.1 \pm 4.1$ & $-41.7 \pm 4.4$ \\
$\mathrm{ClO}_{4}^{-}$ & $-82.5 \pm 7.1$ & $-77.0 \pm 9.1$ & & $-18.8 \pm 1.8$ & $-16.2 \pm 2.1$ \\
$\mathrm{CN}^{-}$ & $-105.6 \pm 8.9$ & $-105.4 \pm 8.8$ & & $-25.0 \pm 2.6$ & $-23.8 \pm 2.2$ \\
$\mathrm{OCN}^{-}$ & $-110.4 \pm 8.2$ & $-110.4 \pm 8.0$ & & $-29.9 \pm 3.0$ & $-29.3 \pm 2.9$ \\
$\mathrm{SCN}^{-}$ & $-92.2 \pm 6.9$ & $-83.5 \pm 9.5$ & & $-24.6 \pm 2.2$ & $-22.4 \pm 3.5$ \\
$\mathrm{NO}_{2}^{-}$ & $-112.7 \pm 8.3$ & $-110.5 \pm 10.3$ & & $-34.5 \pm 3.2$ & $-33.0 \pm 3.5$ \\
$\mathrm{NO}_{3}^{-}$ & $-106.2 \pm 7.6$ & $-106.2 \pm 7.9$ & & $-29.9 \pm 3.2$ & $-29.5 \pm 3.3$
\end{tabular}

${ }^{a}$ Uncertainties represent one standard deviation.

${ }^{b}$ These data are plotted in Fig. 5(a).

${ }^{c}$ These data are plotted in Fig. 7(b).

Table 2. Ensemble-averaged energy components for $\mathrm{ClO}_{n}^{-}(\mathrm{aq})$ in bulk water computed at the SAPT0 + MBD/6-311+G(d,p) level of theory.

\begin{tabular}{lcccccc}
\hline Ion & $\begin{array}{c}\text { Dipole } \\
\text { Moment (D) }\end{array}$ & \multicolumn{5}{c}{ Energy Components (kcal/mol) } \\
\cline { 3 - 7 } & 3.04 & -126.7 & -137.6 & 90.2 & -47.0 & -32.2 \\
\hline $\mathrm{ClO}_{\text {int }}^{-}$ & \rangle$^{b}$ & $\left\langle E_{\text {elst }}\right\rangle$ & $\left\langle E_{\text {exch }}\right\rangle$ & $\left\langle E_{\text {ind }}\right\rangle$ & $\left\langle E_{\text {disp }}\right\rangle$ \\
$\mathrm{ClO}_{2}^{-}$ & 3.20 & -136.2 & -149.6 & 103.6 & -52.3 & -37.8 \\
$\mathrm{ClO}_{3}^{-}$ & 2.46 & -85.8 & -120.6 & 125.1 & -42.1 & -48.2 \\
$\mathrm{ClO}_{4}^{-}$ & 0.00 & -82.5 & -78.1 & 41.1 & -18.8 & -26.7 \\
\hline
\end{tabular}

${ }^{a} \omega \mathrm{B} 97 \mathrm{X}-\mathrm{V} / 6-311+\mathrm{G}(\mathrm{d})$ level of theory at the optimized gas-phase geometry, with the center of nuclear charge as the origin.

${ }^{b}\left\langle E_{\text {int }}\right\rangle=\left\langle E_{\text {elst }}\right\rangle+\left\langle E_{\text {exch }}\right\rangle+\left\langle E_{\text {ind }}\right\rangle+\left\langle E_{\text {disp }}\right\rangle$, up to roundoff error in the averaging.

The chlorate $\left(\mathrm{ClO}_{3}^{-}\right)$ion represents the lone exception to an otherwise systematic correlation between classical and quantum-chemical interaction energies. This particular species is much more strongly solvated by AMOEBA $\left(\left\langle E_{\text {int }}\right\rangle=-126.6 \pm 9.9 \mathrm{kcal} / \mathrm{mol}\right.$ in bulk water $)$ than it is by SAPT0 $+\operatorname{MBD}\left(\left\langle E_{\text {int }}\right\rangle=-85.8 \pm 10.0 \mathrm{kcal} / \mathrm{mol}\right)$. Considering the chlorine oxyanions as a group, the trend amongst the AMOEBA values of $\left|\left\langle E_{\text {int }}\right\rangle\right|$ is $\mathrm{ClO}_{2}^{-}>\mathrm{ClO}_{3}^{-} \gtrsim \mathrm{ClO}^{-} \gg \mathrm{ClO}_{4}^{-}$. The fact that perchlorate $\left(\mathrm{ClO}_{4}^{-}\right)$is an outlier is easy to rationalize in terms of its tetrahedral symmetry and vanishing dipole moment, but the trend amongst the other three chlorine oxyanions is more puzzling. Ensemble-averaged SAPT0 + MBD energy components for the four species $\mathrm{ClO}_{n}^{-}(\mathrm{aq})$ are listed in Table 2, and it is seen that $\left\langle E_{\text {int }}\right\rangle,\left\langle E_{\text {elst }}\right\rangle$, and $\left\langle E_{\text {ind }}\right\rangle$ all follow the same trend exhibited by the gas-phase dipole moments of the ions in question. However, this means that the trend amongst total interaction energies is different from that predicted by AMOEBA. Instead, for the quantum-mechanical calculations the trend (from strongly to weakly interacting) is $\mathrm{ClO}_{2}^{-}>\mathrm{ClO}^{-} \gg \mathrm{ClO}_{3}^{-} \gtrsim \mathrm{ClO}_{4}^{-}$.

In contrast to the AMOEBA results, the SAPT0 + MBD calculations afford similar ensemble-averaged interaction energies for both $\mathrm{ClO}_{3}^{-}$and $\mathrm{ClO}_{4}^{-}$, meaning that the $\mathrm{ClO}_{3}^{-}$ value seems anomalously small, given that all of the chlorine oxyanions except for $\mathrm{ClO}_{4}^{-}$ has a sizable dipole moment. As a sanity check, we recomputed interaction energies for all of the ions using SAPT0(KS) + MBD, which includes intramolecular electron correlation. These results are plotted in Fig. 6(a), which should be compared to the corresponding SAPT0 + MBD results in Fig. 5(a). Total interaction energies at either level of theory are 

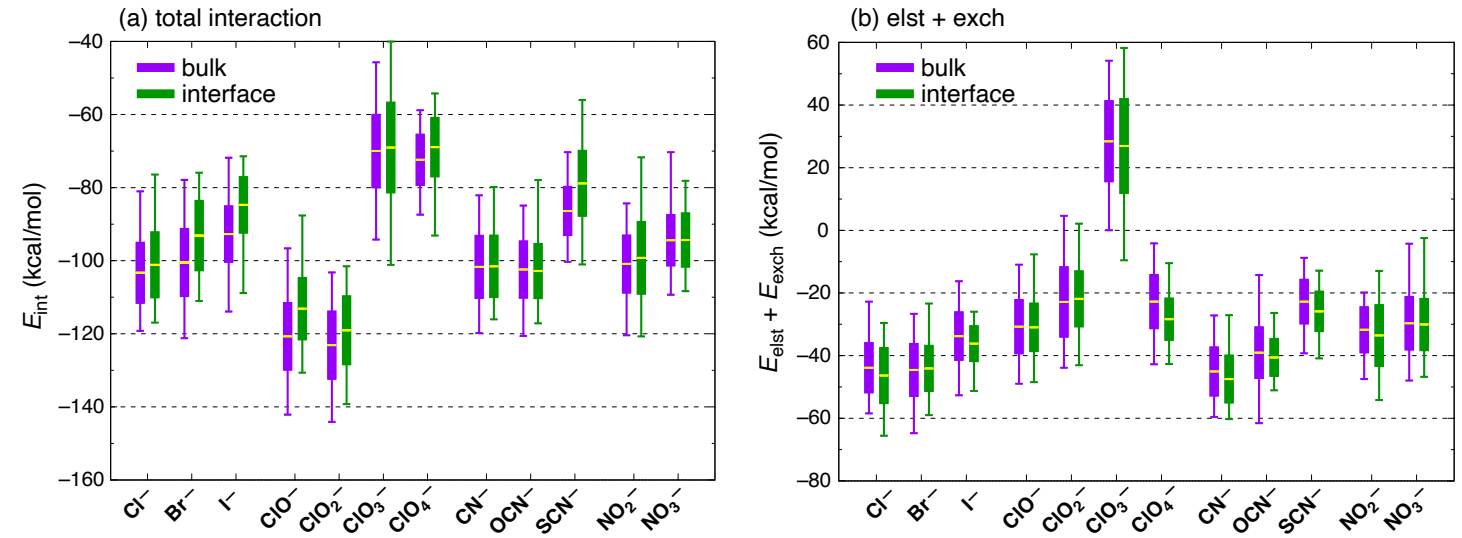

Figure 6. (a) Total interaction energies $\left(E_{\text {int }}\right)$ and (b) first-order electrostatics plus exchange $\left(E_{\text {elst+exch }}\right)$ for ion-water clusters, computed at the SAPT0(KS) +MBD/6-311+G(d,p) level. Panel (a) should be compared to Fig. 5(a), as the difference lies solely in whether HF or KS molecular orbitals are used within the SAPT0 formalism, and the vertical scales are the same in both figures. Similarly, panel (b) should be compared to Fig. 7(a) although the vertical scales are slightly different.
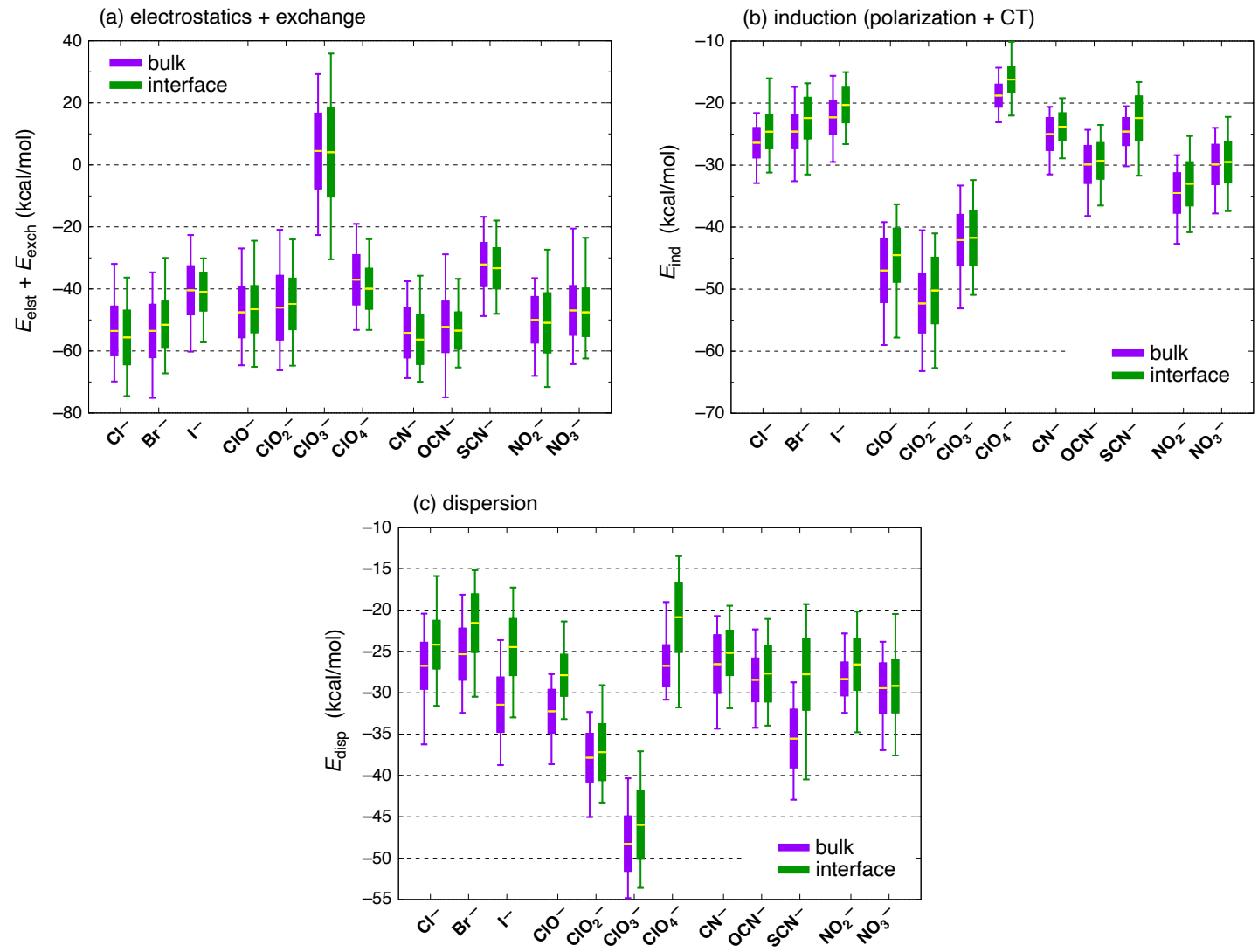

Figure 7. Ion-water interaction energy components computed at the SAPT0 +MBD/6-311+G(d,p) level: (a) first-order electrostatics and exchange, $E_{\text {elst }}+E_{\text {exch }}$ (b) $E_{\text {ind }}$ from Eq. (4); and (c) $E_{\text {disp }}$ from the MBD model. The sum of the energy components in panels (a), (b), and (c) equals $E_{\text {int }}$ in Fig. 5(a). 
quite comparable, and in particular both methods exhibit the same trend amongst the $\mathrm{ClO}_{n}^{-}$ ions, which differs from the trend predicted by AMOEBA.

To investigate this further, we consider the SAPT0 + MBD energy components. These are plotted for each of the ions in Fig. 7, again separating bulk and interfacial environments and ensemble-averaging over either data set. In considering the energy decomposition in Eq. (1), we have opted to group first-order electrostatics and exchange together,

$$
E_{\text {elst+exch }}=E_{\text {elst }}+E_{\text {exch }},
$$

because their sum approximates the electrostatic interaction between antisymmetrized monomer wave functions. This combination of "primitive" electrostatics $\left(E_{\text {elst }}\right.$, which is the Coulomb interaction between isolated-monomer charge densities) and Pauli repulsion $\left(E_{\text {exch }}\right)$ has proven to be easier to interpret for halide-water systems as compared to electrostatics alone $[48,64]$. An example can be found in the ensemble-averaged energy components for the $\mathrm{ClO}_{n}^{-}$(aq) species (Table 2), where the much less repulsive value of $\left\langle E_{\text {exch }}\right\rangle$ for perchlorate at first seems at odds with the larger size of this ion. However, the reduced Pauli repulsion in this case is actually commensurate with a much less attractive value of $\left\langle E_{\text {elst }}\right\rangle$, suggesting a hydration sphere that is not as tight around the ion as it is in smaller (but electrostatically much more attractive) $\mathrm{ClO}_{n}^{-}$ions.

Statistical distributions of $E_{\text {elst+exch }}$ are shown in Fig. 7(a) for all of the ions, and immediately $\mathrm{ClO}_{3}^{-}$stands out as the only ion for which $\left\langle E_{\text {elstexch }}\right\rangle>0$, meaning that the sum of first-order interactions is net repulsive in this case but is net attractive for each of the other ions. These observations are independent of whether one considers the bulk or interfacial data sets because differences between the bulk and interfacial mean values of $E_{\text {elst+exch }}$ are tiny in comparison to the instantaneous fluctuations, as was the case for $E_{\text {int }}$. Furthermore, this anomalous prediction regarding $\mathrm{ClO}_{3}^{-}$is not unique to the SAPT0 level of theory that is used in Fig. 7. A similar anomaly is evident in the SAPTO(KS) results, which can been seen from the statistical distributions of $E_{\text {elst+exch }}$ at that level of theory [Fig. 6(b)]. We note that the largest values of $E_{\text {exch }}$ often correspond to the largest (most attractive) total interaction energies, as is seen for example in SAPT calculations of $\mathrm{ClO}_{n}^{-} \cdots \mathrm{C}_{6} \mathrm{H}_{6}$ complexes $(n=1,2,3,4)$ [84]. In the present case, $\mathrm{ClO}_{3}^{-}$bucks this trend, according to the energy components listed in Table 2.

A possible explanation for the apparently anomalous behavior of $\mathrm{ClO}_{3}^{-}$can be found by examining radial distribution functions (RDFs), $g(r)$, obtained from the MD simulations. (These can be found in the Supporting Information for Ref. 6 but the salient details are described here.) Amongst the chlorine oxyanions, a unique feature of $\mathrm{ClO}_{3}^{-}$is the appearance of two distinct peaks in the RDF for $\mathrm{Cl} \cdots \mathrm{O}_{\mathrm{w}}$ (where $\mathrm{O}_{\mathrm{w}}$ denotes water oxygen), one at $r \approx 3.5 \AA$ and another at $r \approx 4.1 \AA$. For each of the other $\mathrm{ClO}_{n}^{-}$species, the RDF consists of a single well-resolved feature at $r \approx 3.5-3.7 \AA$. The shorter- $r$ feature for $\mathrm{ClO}_{3}^{-}$does not appear to be present in simulations based on a hybrid quantum mechanics/molecular mechanics $(\mathrm{QM} / \mathrm{MM})$ formalism, which were used to interpret $\mathrm{x}$-ray scattering results [85]. If the small- $r$ feature for $\mathrm{ClO}_{3}^{-}$is an indication of an extraneous water molecule present at short range, then this could explain the anomalously repulsive values of $E_{\text {elst+exch }}$ that we then compute at snapshots extracted from the MD simulations. The presence of such a water molecule in those simulations, however, suggests that something in AMOEBA's ion-water interaction is compensating for the short-range repulsion, or perhaps that the latter is simply not repulsive enough. Although polyvalent anions are not considered in the present work (because they are excluded from the air/water interface), it is notable that a short- $r$ peak in the $\mathrm{S} \cdots \mathrm{O}_{\mathrm{w}} \mathrm{RDF}$ is also observed in the simulations of $\mathrm{SO}_{3}^{2-}(\mathrm{aq})$ that were reported in Ref. 6. These feature also appears to be absent from QM/MM simulations and $x$-ray scattering experiments [86]. In view of this, AMOEBA parameterizations for both of these ions ought to be revisited. This is beyond the scope of the present work, though it is interesting to note the way that SAPT analysis of ion-water clusters was able to detect an anomaly. Notably, vertical ionization energies computed for $\mathrm{ClO}_{3}^{-}(\mathrm{aq})$ and $\mathrm{SO}_{3}^{2-}$ (aq) in Ref. 6 are no less accurate, as compared to experimental values [33], than what we obtain 
Table 3. Dispersion energies for $\mathrm{ClO}_{n}^{-}(\mathrm{aq})$ computed using different models and averaged over the bulk and interfacial data sets.

\begin{tabular}{llcc}
\hline System & \multicolumn{2}{c}{$\left\langle E_{\text {disp }}\right\rangle(\mathbf{k c a l} / \mathbf{m o l})^{a}$} \\
\cline { 3 - 4 } & & aiD3 & $\mathbf{M B D}^{b}$ \\
\hline $\mathrm{ClO}^{-}$ & bulk & $-28.0 \pm 2.3$ & $-32.2 \pm 2.6$ \\
$\mathrm{ClO}^{-}$ & interface & $-24.4 \pm 2.2$ & $-27.9 \pm 2.5$ \\
$\mathrm{ClO}_{2}^{-}$ & bulk & $-35.3 \pm 2.8$ & $-37.8 \pm 2.9$ \\
$\mathrm{ClO}_{2}^{-}$ & interface & $-34.8 \pm 3.2$ & $-37.2 \pm 3.4$ \\
$\mathrm{ClO}_{3}^{-}$ & bulk & $-49.7 \pm 3.8$ & $-48.2 \pm 3.3$ \\
$\mathrm{ClO}_{3}^{-}$ & interface & $-47.5 \pm 4.4$ & $-46.0 \pm 4.1$ \\
$\mathrm{ClO}_{4}^{-}$ & bulk & $-28.4 \pm 2.6$ & $-26.7 \pm 2.5$ \\
$\mathrm{ClO}_{4}^{-}$ & interface & $-22.7 \pm 4.5$ & $-20.9 \pm 4.2$ \\
\hline
\end{tabular}

${ }^{a}$ Uncertainties represent one standard deviation.

${ }^{b}$ Based on HF monomer wave functions.

for other inorganic anions including other $\mathrm{ClO}_{n}^{-}$ions [6]. The typical accuracy reported in Ref. 6 is $\sim 0.2 \mathrm{eV}$, considerably smaller than the widths of the corresponding photoelectron spectra.

Returning exclusively to the monovalent ions and examining the other energy components whose statistics are summarized in Fig. 7, another curiosity arises in regard to dispersion energies for the chlorine oxyanions. Dispersion is size-extensive, so that all else being equal it should scale in proportion to the number of electrons. For the $\mathrm{ClO}_{n}^{-}$species, however, we observe that $\left|E_{\text {disp }}\right|$ decreases in the order $\mathrm{ClO}_{3}^{-}>\mathrm{ClO}_{2}^{-}>\mathrm{ClO}^{-}>\mathrm{ClO}_{4}^{-}$. This time, perchlorate is the apparent anomaly. Dispersion energies in Fig. 7(c) were computed using the MBD model [46], so as a sanity check we recomputed $E_{\text {disp }}$ using the third-generation $a b$ initio dispersion potential aiD3 [34], which consists of atom-atom $C_{6}$ and $C_{8}$ potentials fitted to dispersion-only data from high-level SAPT calculations. Dispersion energies obtain for the $\mathrm{ClO}_{n}^{-}$species with both dispersion models are provided in Table 3 as ensemble averages. Both models afford rather similar dispersion energies, consistent with previous tests for cases where many-body effects on $E_{\text {disp }}$ are not significant [46]. (In the context of dispersion, "many-body" implies an effect that cannot be described by pairwise atom-atom potentials [44,87]. These typically arise in conjugated molecules where screening effects significantly modify the effective $C_{6}$ coefficients [88]. For small molecules, three-body dispersion effects are quite small [74].) Notably, in the aiD3 model the $C_{6}$ and $C_{8}$ coefficients depend only on atomic number and do not respond to the electronic structure of the monomers.

The sharp drop in dispersion between chlorate $\left(\mathrm{ClO}_{3}^{-}\right)$and perchlorate is a feature of both dispersion models, suggesting that this is not an artifact. A likely explanation is that in perchlorate, the addition of a fourth oxygen atom around the central (and more polarizable) chlorine atom screens the water molecules from this polarizable center, and thus significantly attenuates chlorine's contribution to the dispersion energy. In contrast, for the other $\mathrm{ClO}_{n}^{-}$ions the chlorine atom remains solvent-exposed and the dispersion is much larger. This mechanism would be reflected in both dispersion models, if only as a function of increased chlorine-water distance in the aiD3 case. Also in support of this hypothesis are the data in Fig. 7(b) for SAPT0 + MBD induction energies, which also exhibit a pronounced drop in magnitude between $\mathrm{ClO}_{3}^{-}$and $\mathrm{ClO}_{4}^{-}$. As compared to dispersion interactions, polarization effects decay more slowly with distance, e.g., as $r^{-4}$ for charge-dipole polarization, but this dependence is still rather steep.

Polarization is often invoked in discussions of ions at the air/water interface [8-16], so it is interesting to note that induction energies are systematically smaller in the interfacial environment [Fig. 7(b)]. As with the total interaction energies, however, the difference between bulk and interfacial mean values $\left\langle E_{\text {ind }}\right\rangle$ is small in comparison to the instantaneous fluctuations as measured by the standard deviation. The numerical data corresponding to Fig. 7(b) are provided in Table 1. Note that "polarization" as it is typically understood 

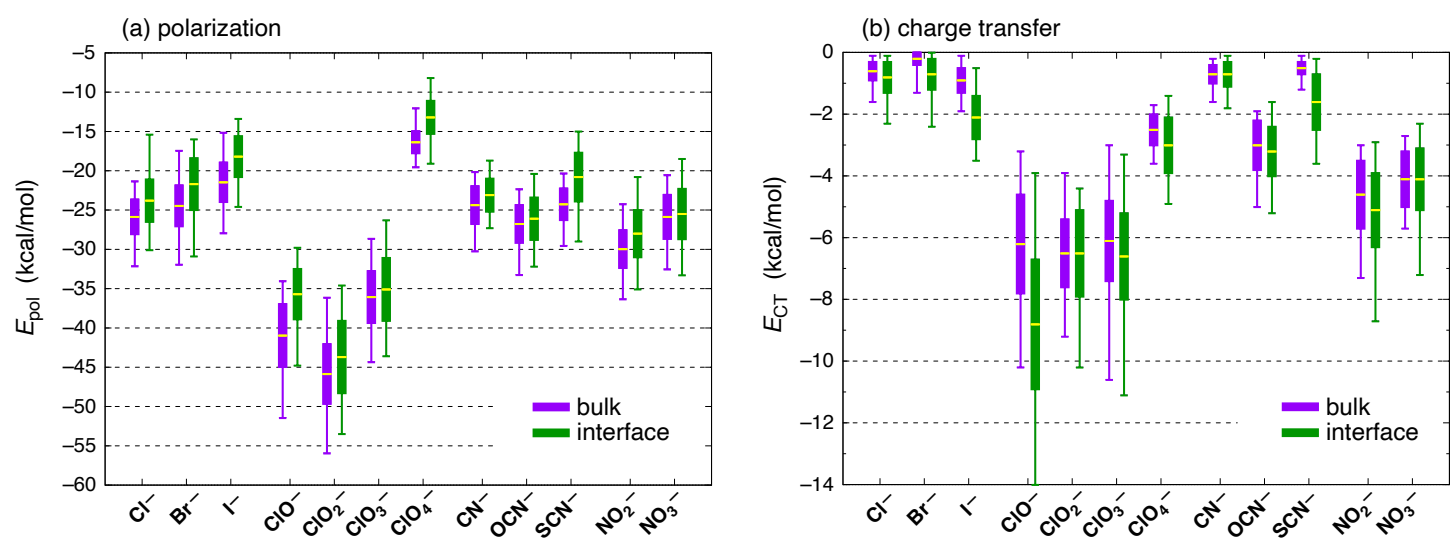

Figure 8. (a) Polarization energies $\left(E_{\mathrm{pol}}\right)$ and (b) CT energies $\left(E_{\mathrm{CT}}\right)$, computed at the SAPT0 $+\mathrm{MBD} / 6-311+\mathrm{G}(\mathrm{d}, \mathrm{p})$ level. Together, $E_{\text {pol }}+E_{\mathrm{CT}}=E_{\text {ind }}$, where the sum (induction energy) is plotted in Fig. 7(b).

means strictly intramolecular redistribution of charge, with CT considered as a separate effect, and these two parts of the induction energy are separated in Fig. 8. Because the CT-free polarization energy $\left(E_{\mathrm{pol}}\right)$ is much larger than the CT energy $\left(E_{\mathrm{CT}}\right)$, the result is that $E_{\text {pol }}$ follows essentially the same trend from ion to ion as does the total induction energy, $E_{\text {ind }}$. In particular, this means that the polarization energy is systematically smaller in the interfacial environment, for each of the ions considered here. Indeed, for the canonical surface-active anions $\mathrm{Br}^{-}, \mathrm{I}^{-}, \mathrm{ClO}_{4}^{-}$and $\mathrm{SCN}^{-}[18,31,32,89]$, the polarization energy is significantly smaller in the interfacial environment, by at least the standard deviation of $E_{\text {pol }}$ in bulk water; see Fig. 8(a).

That observation, in turn, is a direct result of $\mathrm{CT}$ energies that are systematically larger at the interface for precisely those four surface-active anions. Statistical distributions of $E_{\mathrm{CT}}$ for all of the ions are plotted in Fig. 8(b), and in contrast to other energy components, only for $E_{\mathrm{CT}}$ do we observe pronounced difference between averages computed for the bulk and interfacial data sets. That said, the overall scale of the CT energies is a rather small part of either the total induction energy or the total interaction energy, with $\left|E_{\mathrm{CT}}\right| \lesssim 10 \mathrm{kcal} / \mathrm{mol}$ except in the case of interfacial $\mathrm{ClO}^{-}$. (Although $\mathrm{CT}$ energies smaller than $10 \mathrm{kcal} / \mathrm{mol}$ do play a pivotal role in establishing the directionality of hydrogen bonds [48,64], that kind of detailed analysis of a potential energy surface is not attempted in the present work, where we are interested in ensemble-averaged properties.) $\mathrm{For} \mathrm{Br}^{-}, \mathrm{I}^{-}, \mathrm{ClO}_{4}^{-}$, and $\mathrm{SCN}^{-}$, the average $\mathrm{CT}$ energy at the air/water interface is larger than its mean value in bulk water by at least one standard deviation in the bulk value. The $\mathrm{Cl}^{-}$ion exhibits an interfacial value of $\left\langle E_{\mathrm{CT}}\right\rangle$ that is not quite one standard deviation larger than its mean value in bulk water.

In the context of the Hofmeister series [90,91], the anions $\mathrm{I}^{-}, \mathrm{ClO}_{4}^{-}$, and $\mathrm{SCN}^{-}$have especially large binding constants to protein [91,92], which is historically associated with the definition of chaotropes or "structure breakers" [7], whereas $\mathrm{Cl}^{-}$binds more weakly [92]. That said, $\mathrm{NO}_{3}^{-}$is usually categorized as a structure-breaker on par with $\mathrm{Br}^{-}$in the Hofmeister series [90], and as weakly surface-active on the basis of VSFG measurements [18], yet the mean values of $E_{\mathrm{CT}}$ that we obtain for $\mathrm{NO}_{3}^{-}$are nearly identical in the bulk and interfacial environments. The hypochlorite ion $\left(\mathrm{ClO}^{-}\right)$is an outlier in our $\mathrm{CT}$ analysis, with a significantly larger value of $\left|E_{C T}\right|$ at the air/water interface, but this species is not typically discussed in the context of the Hofmeister series or VSFG studies of the air/water interface due to its limited stability in aqueous solution.

\section{Conclusions}

Detailed analysis of anion-water clusters extracted from MD simulations of the air/ water interface reveals that the total ion-water interaction energy (considering two solvation shells around the ion) is systematically larger for a given ion in bulk water than it is for the same ion near the interface. The same is true for the CT-free polarization component of 
the total interaction energy, which is interesting given that polarization is often assumed to play a central role in surface activity [12]. The aforementioned conclusions hold equally well for "soft" (i.e., low surface charge density) anions that are considered surface active $\left(\mathrm{Br}^{-}, \mathrm{I}^{-}, \mathrm{ClO}_{4}^{-}\right.$, and $\left.\mathrm{SCN}^{-}\right)$, as well as for hard anions that are not considered surface active $\left(\mathrm{CN}^{-}, \mathrm{OCN}^{-}\right.$, and $\left.\mathrm{NO}_{2}^{-}\right)$. However, systematic differences in the mean values $\left\langle E_{\mathrm{int}}\right\rangle$ and $\left\langle E_{\mathrm{pol}}\right\rangle$ in bulk versus interfacial environments are rather small in comparison to the magnitude of the instantaneous fluctuations in $E_{\mathrm{int}}$ and $E_{\mathrm{pol}}$. Anion-to-water CT stands out as the only energy component whose magnitude is larger at the interface for some ions, and in fact it is larger specifically for the traditional surface-active anions: $\mathrm{Br}^{-}, \mathrm{I}^{-}$, $\mathrm{ClO}_{4}^{-}$, and $\mathrm{SCN}^{-}$. However, $\mathrm{NO}_{3}^{-}$can also be detected in surface-sensitive vibrational spectroscopy [18], yet for that species $\left\langle E_{\mathrm{CT}}\right\rangle$ is essentially the same at the interface as it is in bulk water. In all cases, the CT energy constitutes less than $20 \%$ of the total induction energy, meaning that it is at least $5 \times$ smaller than the CT-free polarization energy. The latter does not exhibit a surface preference and is in fact larger in bulk water.

Considering the halide ions as a series that ranges from kosmotropic to chaotropic [7], or equivalently whose surface activities decrease in the order $\mathrm{I}^{-}>\mathrm{Br}^{-}>\mathrm{Cl}^{-} \gg \mathrm{F}^{-}$, it has previously been noted that no single mechanistic explanation for this ordering can be gleaned from atomistic simulations [20]. Changes in the water-water interactions as the surface-active ions approach the air/water interface appears to play a role [20]. The present analysis, based on accurate quantum-mechanical calculations of ion-water interaction energies, supports the notion that ion-water interactions alone do not readily afford any kind of a diagnostic (let alone a mechanism) to determine whether an ion resides in a bulk or interfacial environment. This null result complements our recent conclusion that short-range (first-shell) solvation structure is extremely similar in the bulk and interfacial environments [6]. The detailed mechanism of soft anion surface activity remains an open question.

Author Contributions: Conceptualization, J.M.H; methodology, J.M.H.; software, J.M.H. and S.K.P.; validation, J.M.H.; formal analysis, J.M.H.; investigation, J.M.H. and S.K.P.; resources, J.M.H.; data curation, J.M.H.; writing — original draft preparation, J.M.H.; writing — review and editing, J.M.H. and S.KP.; visualization, J.M.H.; supervision, J.M.H.; project administration, J.M.H.; funding acquisition, J.M.H.. All authors have read and agreed to the published version of the manuscript.

Funding: This research was funded by the U.S. National Science Foundation under grant number CHE-1955282.

Institutional Review Board Statement: Not applicable.

Informed Consent Statement: Not applicable.

Data Availability Statement: The data that support this study are available from the corresponding author upon reasonable request.

Acknowledgments: It is a pleasure to wish Frank Weinhold a happy 80th birthday. J.M.H. thanks Frank for his excellent teaching when the author was a graduate student at the University of Wisconsin.

Conflicts of Interest: J.M.H. serves on the board of directors of Q-Chem Inc.

\section{References}

1. Verreault, D.; Hua, W.; Allen, H.C. From conventional to phase-sensitive vibrational sum frequency generation spectroscopy: Probing water organization at aqueous interfaces. J. Phys. Chem. Lett. 2012, 3, 3012-3028. doi:10.1021/jz301179g.

2. Raymond, E.A.; Richmond, G.L. Probing the molecular structure and bonding of the surface of aqueous salt solutions. J. Phys. Chem. B 2004, 108, 5051-5059. doi:10.1021/jp037725k.

3. Liu, D.; Ma, G.; Levering, L.M.; Allen, H.C. Vibrational spectroscopy of aqueous sodium halide solutions and air-liquid interfaces: Observation of increased interfacial depth. J. Phys. Chem. B 2004, 108, 2252-2260. doi:10.1021/jp036169r.

4. Mucha, M.; Frigato, T.; Levering, L.M.; Allen, H.C.; Tobias, D.J.; Dang, L.X.; Jungwirth, P. Unified molecular picture of the surfaces of aqueous acid, base, and salt solutions. J. Phys. Chem. B 2005, 109, 7617-7623. doi:10.1021/jp0445730.

5. Garrett, B. Ions at the air/water interface. Science 2004, 303, 1146-1147. doi:10.1126/science.1089801. 
6. Paul, S.K.; Herbert, J.M. Probing interfacial effects on ionization energies: The surprising banality of anion-water hydrogen bonding at the air/water interface. J. Am. Chem. Soc. 2021, 143, 10189-10202. doi:10.1021/jacs.1c03131.

7. Collins, K.D. Charge density-dependent strength of hydration and biological structure. Biophys. J. 1997, 72, 65-76. doi:10.1016/S0006-3495(97)78647-8.

8. Jungwirth, P.; Tobias, D.J. Ions at the air/water interface. J. Phys. Chem. B 2002, 106, 6361-6373. doi:10.1021/jp020242g.

9. Dang, L.X. Computational study of ion binding to the liquid interface of water. J. Phys. Chem. B 2002, 106, 10388-10394. doi:10.1021/jp021871t.

10. Herce, D.H.; Perera, L.; Darden, T.A.; Sagui, C. Surface solvation for an ion in a water cluster. J. Chem. Phys. 2005, 122, 024513:1-10. doi:10.1063/1.1829635.

11. Archontis, G.; Leontidis, E.; Andreou, G. Attraction of iodide ions by the free water surface, revealed by simulations with a polarizable force field based on Drude oscillators. J. Phys. Chem. B 2005, 109, 17957-17966. doi:10.1021/jp0526041.

12. Jungwirth, P.; Tobias, D.J. Specific ion effects at the air/water interface. Chem. Rev. 2006, 106, 1259-1281. doi:10.1021/cr0403741.

13. Chang, T.M.; Dang, L.X. Recent advances in molecular simulations of ion solvation at liquid interfaces. Chem. Rev. 2006, 106, 1305-1322. doi:10.1021/cr0403640.

14. Ishiyama, T.; Morita, A. Molecular dynamics study of gas-liquid aqueous sodium halide interfaces. I. Flexible and polarizable molecular modeling and interfacial properties. J. Phys. Chem. C 2007, 111, 721-737. doi:10.1021/jp065191s.

15. Dweik, J.; Srour, M.; Karaky, K.; Kobeissi, M.; Joumaa, W.; Abou-Saleh, K. Molecular simulation of ion transport at the water/ vapor interface. Open J. Phys. Chem. 2012, 2, 147-155. doi:10.4236/ojpc.2012.23020.

16. Sun, L.; Li, X.; Tu, Y.; Ågren, H. Origin of ion selectivity at the air/water interface. Phys. Chem. Chem. Phys. 2015, 17, 4311-4318. doi:10.1039/c4cp03338h.

17. Jungwirth, P.; Curtis, J.E.; Tobias, D.J. Polarizability and aqueous solvation of the sulfate dianion. Chem. Phys. Lett. 2003, 367, 704-710. doi:10.1016/S0009-2614(02)01782-7.

18. Jubb, A.M.; Hua, W.; Allen, H.C. Environmental chemistry at vapor/water interfaces: Insights from vibrational sum frequency generation spectroscopy. Annu. Rev. Phys. Chem. 2012, 63, 107-130. doi:10.1146/annurev-physchem-032511-143811.

19. Jungwirth, P.; Tobias, D.J. Molecular structure of salt solutions: A new view of the interface with implications for heterogeneous atmospheric chemistry. J. Phys. Chem. B 2001, 105, 10468-10472. doi:10.1021/jp012750g.

20. Caleman, C.; Hub, J.S.; van Maaren, P.J.; van der Spoel, D. Atomistic simulation of ion solvation in water explains surface preference of halides. Proc. Natl. Acad. Sci. USA 2011, 108, 6838-6842. doi:10.1073/pnas.1017903108.

21. Petersen, P.B.; Saykally, R.J.; Mucha, M.; Jungwirth, P. Enhanced concentration of polarizable anions at the liquid water surface: SHG spectroscopy and MD simulations of sodium thiocyanide. J. Phys. Chem. B 2005, 109, 10915-10921. Erratum: ibid. 109, 13402 (2005), doi:10.1021/jp050864c.

22. Baer, M.D.; Mundy, C.J. Toward an understanding of the specific ion effect using density functional theory. J. Phys. Chem. Lett. 2011, 2, 1088-1093. doi:10.1021/jz200333b.

23. Netz, R.R.; Horinek, D. Progress in modeling of ion effects at the vapor/water interface. Annu. Rev. Phys. Chem. 2012, 63, 401-418. doi:10.1146/annurev-physchem-032511-143813.

24. Ou, S.; Hu, Y.; Patel, S.; Wan, H. Spherical monovalent ions at aqueous liquid-vapor interfaces: Interfacial stability and induced interface fluctuations. J. Phys. Chem. B 2013, 117, 11732-11742. doi:10.1021/jp406001b.

25. Ou, S.C.; Cui, D.; Patel, S. Molecular modeling of ions at interfaces: Exploring similarities to hydrophobic solvation through the lens of induced aqueous interfacial fluctuations. Phys. Chem. Chem. Phys. 2016, 18, 30357-30365. doi:10.1039/C6CP04112D.

26. Ishiyama, T.; Imamura, T.; Morita, A. Theoretical studies of structures and vibrational sum frequency generation spectra at aqueous interfaces. Chem. Rev. 2014, 114, 8447-8470. doi:10.1021/cr4004133.

27. Levin, Y.; dos Santos, A.P.; Diehl, A. Ions at the air-water interface: An end to a hundred-year-old mystery? Phys. Rev. Lett. 2009, 103, 257802:1-4. doi:10.1103/PhysRevLett.103.257802.

28. Levin, Y.; dos Santos, A.P. Ions at hydrophobic interfaces. J. Phys.: Condens. Matt. 2014, 26, 203101:1-11. doi:10.1088/0953$8984 / 26 / 20 / 203101$.

29. Wick, C.D.; Xantheas, S.S. Computational investigation of the first solvation shell structure of interfacial and bulk aqueous chloride and iodide ions. J. Phys. Chem. B 2009, 113, 4141-4146. doi:10.1021/jp806782r.

30. Baer, M.D.; Mundy, C.J. An ab initio approach to understanding the specific ion effect. Faraday Discuss. 2013, 160, 89-101. doi:10.1039/C2FD20113E.

31. Viswanath, P.; Motschmann, H. Oriented thiocyanate anions at the air-electrolyte interface and its implications on interfacial water-a vibrational sum frequency spectroscopy study. J. Phys. Chem. C 2007, 111, 4484-4486. doi:10.1021/jp0702649.

32. Viswanath, P.; Motschmann, M. Effect of interfacial presence of oriented thiocyanate on water structure. J. Phys. Chem. C 2008, 112, 2099-2103. doi:10.1021/jp077229m.

33. Seidel, R.; Winter, B.; Bradforth, S.E. Valence electronic structure of aqueous solutions: Insights from photoelectron spectroscopy. Annu. Rev. Phys. Chem. 2016, 67, 283-305. doi:10.1146/annurev-physchem-040513-103715.

34. Lao, K.U.; Herbert, J.M. Accurate and efficient quantum chemistry calculations of noncovalent interactions in many-body systems: The XSAPT family of methods. J. Phys. Chem. A 2015, 119, 235-253. doi:10.1021/jp5098603.

35. Carter-Fenk, K.; Lao, K.U.; Herbert, J.M. Predicting and understanding non-covalent interactions using novel forms of symmetryadapted perturbation theory. Acc. Chem. Res. 2021, 54, 3679-3690. doi:10.1021/acs.accounts.1c00387. 
36. Francisco, E.; Pendás, A.M. Energy partition analyses: Symmetry-adapted perturbation theory and other techniques. In NonCovalent Interactions in Quantum Chemistry and Physics; de la Roza, A.O.; DiLabio, G.A., Eds.; Elsevier: Amsterdam, 2017; chapter 2, pp. 27-64. doi:10.1016/B978-0-12-809835-6.00003-7.

37. Patkowski, K. Recent developments in symmetry-adapted perturbation theory. WIREs Comput. Mol. Sci. 2020, 10, e1452:1-47. doi:10.1002/wcms.1452.

38. Ren, P.; Ponder, J.W. Polarizable atomic multipole water model for molecular mechanics simulation. J. Phys. Chem. B 2003, 107, 5933-5947. doi:10.1021/jp027815\\%2B.

39. Grossfield, A.; Ren, P.; Ponder, J.W. Ion solvation thermodynamics from simulation with a polarizable force field. J. Am. Chem. Soc. 2003, 125, 15671-15682. doi:10.1021/ja037005r.

40. Ren, P.; Wu, C.; Ponder, J.W. Polarizable atomic multipole-based molecular mechanics for organic molecules. J. Chem. Theory Comput. 2011, 7, 3143-3161. doi:10.1021/ct200304d.

41. Rackers, J.A.; Wang, Z.; Lu, C.; Laury, M.L.; Lagardère, L.; Schnieders, M.J.; Piquemal, J.P.; Ren, P.; Ponder, J.W. Tinker 8: Software tools for molecular design. J. Chem. Theory Comput. 2018, 14, 5273-5289. doi:10.1021/acs.jctc.8b00529.

42. Parker, T.M.; Burns, L.A.; Parrish, R.M.; Ryno, A.G.; Sherrill, C.D. Levels of symmetry adapted perturbation theory (SAPT). I. Efficiency and performance for interaction energies. J. Chem. Phys. 2014, 140, 094106:1-16. doi:10.1063/1.4867135.

43. Gray, M.; Herbert, J.M. Simplified tuning of long-range corrected density functionals for symmetry-adapted perturbation theory. J. Chem. Phys. 2021, 155, 034103:1-8. doi:10.1063/5.0059364.

44. Lao, K.U.; Herbert, J.M. Atomic orbital implementation of extended symmetry-adapted perturbation theory (XSAPT) and benchmark calculations for large supramolecular complexes. J. Chem. Theory Comput. 2018, 14, 2955-2978. doi:10.1021/acs.jctc.8b00058.

45. Ambrosetti, A.; Reilly, A.M.; DiStasio Jr., R.A.; Tkatchenko, A. Long-range correlation energy calculated from coupled atomic response functions. J. Chem. Phys. 2014, 140, 18A508:1-14. doi:10.1063/1.4865104.

46. Carter-Fenk, K.; Lao, K.U.; Liu, K.Y.; Herbert, J.M. Accurate and efficient ab initio calculations for supramolecular complexes: Symmetry-adapted perturbation theory with many-body dispersion. J. Phys. Chem. Lett. 2019, 10, $2706-2714$. doi:10.1021/acs.jpclett.9b01156.

47. Epifanovsky, E.; Gilbert, A.T.B.; Feng, X.; Lee, J.; Mao, Y.; Mardirossian, N.; Pokhilko, P.; White, A.F.; Coons, M.P.; Dempwolff, A.L.; Gan, Z.; Hait, D.; Horn, P.R.; Jacobson, L.D.; Kaliman, I.; Kussmann, J.; Lange, A.W.; Lao, K.U.; Levine, D.S.; Liu, J.; McKenzie, S.C.; Morrison, A.F.; Nanda, K.D.; Plasser, F.; Rehn, D.R.; Vidal, M.L.; You, Z.Q.; Zhu, Y.; Alam, B.; Albrecht, B.J.; Aldossary, A.; Alguire, E.; Andersen, J.H.; Athavale, V.; Barton, D.; Begam, K.; Behn, A.; Bellonzi, N.; Bernard, Y.A.; Berquist, E.J.; Burton, H.G.A.; Carreras, A.; Carter-Fenk, K.; Chakraborty, R.; Chien, A.D.; Closser, K.D.; Cofer-Shabica, V.; Dasgupta, S.; de Wergifosse, M.; Deng, J.; Diedenhofen, M.; Do, H.; Ehlert, S.; Fang, P.T.; Fatehi, S.; Feng, Q.; Friedhoff, T.; Gayvert, J.; Ge, Q.; Gidofalvi, G.; Goldey, M.; Gomes, J.; González-Espinoza, C.E.; Gulania, S.; Gunina, A.O.; Hanson-Heine, M.W.D.; Harbach, P.H.P.; Hauser, A.; Herbst, M.F.; Hernández Vera, M.; Hodecker, M.; Holden, Z.C.; Houck, S.; Huang, X.; Hui, K.; Huynh, B.C.; Ivanov, M.; Jász, A.; Ji, H.; Jiang, H.; Kaduk, B.; Kähler, S.; Khistyaev, K.; Kim, J.; Kis, G.; Klunzinger, P.; Koczor-Benda, Z.; Koh, J.H.; Kosenkov, D.; Koulias, L.; Kowalczyk, T.; Krauter, C.M.; Kue, K.; Kunitsa, A.; Kus, T.; Ladjánszki, I.; Landau, A.; Lawler, K.V.; Lefrancois, D.; Lehtola, S.; Li, R.R.; Li, Y.P.; Liang, J.; Liebenthal, M.; Lin, H.H.; Lin, Y.S.; Liu, F.; Liu, K.Y.; Loipersberger, M.; Luenser, A.; Manjanath, A.; Manohar, P.; Mansoor, E.; Manzer, S.F.; Mao, S.P.; Marenich, A.V.; Markovich, T.; Mason, S.; Maurer, S.A.; McLaughlin, P.F.; Menger, M.F.S.J.; Mewes, J.M.; Mewes, S.A.; Morgante, P.; Mullinax, J.W.; Oosterbaan, K.J.; Paran, G.; Paul, A.C.; Paul, S.K.; Pavošević, F.; Pei, Z.; Prager, S.; Proynov, E.I.; Rák, A.; Ramos-Cordoba, E.; Rana, B.; Rask, A.E.; Rettig, A.; Richard, R.M.; Rob, F.; Rossomme, E.; Scheele, T.; Scheurer, M.; Schneider, M.; Sergueev, N.; Sharada, S.M.; Skomorowski, W.; Small, D.W.; Stein, C.J.; Su, Y.C.; Sundstrom, E.J.; Tao, Z.; Thirman, J.; Tornai, G.J.; Tsuchimochi, T.; Tubman, N.M.; Veccham, S.P.; Vydrov, O.; Wenzel, J.; Witte, J.; Yamada, A.; Yao, K.; Yeganeh, S.; Yost, S.R.; Zech, A.; Zhang, I.Y.; Zhang, X.; Zhang, Y.; Zuev, D.; Aspuru-Guzik, A.; Bell, A.T.; Besley, N.A.; Bravaya, K.B.; Brooks, B.R.; Casanova, D.; Chai, J.D.; Coriani, S.; Cramer, C.J.; Cserey, G.; DePrince III, A.E.; DiStasio Jr., R.A.; Dreuw, A.; Dunietz, B.D.; Furlani, T.R.; Goddard III, W.A.; Hammes-Schiffer, S.; Head-Gordon, T.; Hehre, W.J.; Hsu, C.P.; Jagau, T.C.; Jung, Y.; Klamt, A.; Kong, J.; Lambrecht, D.S.; Liang, W.; Mayhall, N.J.; McCurdy, C.W.; Neaton, J.B.; Ochsenfeld, C.; Parkhill, J.A.; Peverati, R.; Rassolov, V.A.; Shao, Y.; Slipchenko, L.V.; Stauch, T.; Steele, R.P.; Subotnik, J.E.; Thom, A.J.W.; Tkatchenko, A.; Truhlar, D.G.; Van Voorhis, T.; Wesolowski, T.A.; K. B. Whaley, H.L.W.; Zimmerman, P.M.; Faraji, S.; Gill, P.M.W.; Head-Gordon, M.; Herbert, J.M.; Krylov, A.I. Software for the frontiers of quantum chemistry: An overview of developments in the Q-Chem 5 package. J. Chem. Phys. 2021, 155, 084801:1-59. doi:10.1063/5.0055522.

48. Herbert, J.M. Neat, simple, and wrong: Debunking electrostatic fallacies regarding noncovalent interactions. J. Phys. Chem. A 2021, 125, 7125-7137. doi:10.1021/acs.jpca.1c05962.

49. Rohrdanz, M.A.; Martins, K.M.; Herbert, J.M. A long-range-corrected density functional that performs well for both ground-state properties and time-dependent density functional theory excitation energies, including charge-transfer excited states. J. Chem. Phys. 2009, 130, 054112:1-8. doi:10.1063/1.3073302.

50. Lao, K.U.; Herbert, J.M. Symmetry-adapted perturbation theory with Kohn-Sham orbitals using non-empirically tuned, longrange-corrected density functionals. J. Chem. Phys. 2014, 140, 044108:1-8. doi:10.1063/1.4862644.

51. Hapka, M.; Rajchel, L.; Modrzejewski, M.; Chałasiǹski, G.; Szczęśniak, M.M. Tuned range-separated hybrid functionals in the symmetry-adapted perturbation theory. J. Chem. Phys. 2014, 141, 134120:1-10. doi:10.1063/1.4896608.

52. Baer, R.; Livshits, E.; Salzner, U. Tuned range-separated hybrids in density functional theory. Annu. Rev. Phys. Chem. 2010, 61, 85-109. doi:10.1146/annurev.physchem.012809.103321. 
53. Alam, B.; Morrison, A.F.; Herbert, J.M. Charge separation and charge transfer in the low-lying excited states of pentacene. J. Phys. Chem. C 2020, 124, 24653-24666. doi:10.1021/acs.jpcc.0c07932.

54. Uhlig, F.; Herbert, J.M.; Coons, M.P.; Jungwirth, P. Optical spectroscopy of the bulk and interfacial hydrated electron from ab initio calculations. J. Phys. Chem. A 2014, 118, 7507-7515. doi:10.1021/jp5004243.

55. Jacobson, L.D.; Herbert, J.M. An efficient, fragment-based electronic structure method for molecular systems: Self-consistent polarization with perturbative two-body exchange and dispersion. J. Chem. Phys. 2011, 134, 094118:1-17. doi:10.1063/1.3560026.

56. Herbert, J.M.; Jacobson, L.D.; Lao, K.U.; Rohrdanz, M.A. Rapid computation of intermolecular interactions in molecular and ionic clusters: Self-consistent polarization plus symmetry-adapted perturbation theory. Phys. Chem. Chem. Phys. 2012, 14, 7679-7699. doi:10.1039/C2CP24060B.

57. Jacobson, L.D.; Richard, R.M.; Lao, K.U.; Herbert, J.M. Efficient monomer-based quantum chemistry methods for molecular and ionic clusters. Аnпu. Rep. Comput. Chem. 2013, 9, 25-58. doi:10.1016/B978-0-444-62672-1.00002-9.

58. Liu, K.Y.; Carter-Fenk, K.; Herbert, J.M. Self-consistent charge embedding at very low cost, with application to symmetry-adapted perturbation theory. J. Chem. Phys. 2019, 151, 031102:1-7. doi:10.1063/1.5111869.

59. Moszyński, R.; Cybulski, S.M.; Chałasiński, G. Many-body theory of intermolecular induction interactions. J. Chem. Phys. 1994, 100, 4998-5010. doi:10.1063/1.467218.

60. Lao, K.U.; Herbert, J.M. Energy decomposition analysis with a stable charge-transfer term for interpreting intermolecular interactions. J. Chem. Theory Comput. 2016, 12, 2569-2582. doi:10.1021/acs.jctc.6b00155.

61. Kaduk, B.; Kowalczyk, T.; Van Voorhis, T. Constrained density functional theory. Chem. Rev. 2012, 112, 321-370. doi:10.1021/cr200148b.

62. Ǩezáč, J.; de la Lande, A. Robust, basis-set independent method for the evaluation of charge-transfer energy in noncovalent complexes. J. Chem. Theory Comput. 2015, 11, 528-537. doi:10.1021/ct501115m.

63. Ǩezác, J.; de la Lande, A. On the role of charge transfer in halogen bonding. Phys. Chem. Chem. Phys. 2017, 19, 791-803. doi:10.1039/C6CP07475H.

64. Herbert, J.M.; Carter-Fenk, K. Electrostatics, charge transfer, and the nature of the halide-water hydrogen bond. J. Phys. Chem. A 2021, 125, 1243-1256. doi:10.1021/acs.jpca.0c11356.

65. Becke, A.D. A multicenter numerical integration scheme for polyatomic molecules. J. Chem. Phys. 1988, 88, $2547-2553$. doi:10.1063/1.454033.

66. Medvedev, N.N. The algorithm for three-dimensional Voronoi polyhedra. J. Comput. Phys. 1986, 67, 223-229. doi:10.1016/00219991(86)90123-3.

67. Slater, J.C. Atomic radii in crystals. J. Chem. Phys. 1964, 41, 3199-3204. doi:10.1063/1.1725697.

68. Dasgupta, S.; Herbert, J.M. Standard grids for high-precision integration of modern density functionals: SG-2 and SG-3. J. Comput. Chem. 2017, 38, 869-882. doi:10.1002/jcc.24761.

69. Wu, Q.; Chen, C.L.; Van Voorhis, T. Configuration interaction based on constrained density functional theory: A multireference method. J. Chem. Phys. 2007, 127, 164119:1-9. doi:10.1063/1.2800022.

70. Wu, Q.; Van Voorhis, T. Direct optimization method to study constrained systems within density-functional theory. Phys. Rev. A 2005, 72, 024502:1-4. doi:10.1103/PhysRevA.72.024502.

71. Richard, R.M.; Lao, K.U.; Herbert, J.M. Aiming for benchmark accuracy with the many-body expansion. Acc. Chem. Res. 2014, 47, 2828-2836. doi:10.1021/ar500119q.

72. Lao, K.U.; Liu, K.Y.; Richard, R.M.; Herbert, J.M. Understanding the many-body expansion for large systems. II. Accuracy considerations. J. Chem. Phys. 2016, 144, 164105:1-15. doi:10.1063/1.4947087.

73. Liu, K.Y.; Herbert, J.M. Understanding the many-body expansion for large systems. III. Critical role of four-body terms, counterpoise corrections, and cutoffs. J. Chem. Phys. 2017, 147, 161729:1-13. doi:10.1063/1.4986110.

74. Herbert, J.M. Fantasy versus reality in fragment-based quantum chemistry. J. Chem. Phys. 2019, 151, 170901:1-38. doi:10.1063/1.5126216.

75. Heindel, J.P.; Xantheas, S.S. The many-body expansion for aqueous systems revisited: I. Water-water interactions. J. Chem. Theory Comput. 2020, 16, 6843-6855. doi:10.1021/acs.jctc.9b00749.

76. Heindel, J.P.; Xantheas, S.S. The many-body expansion for aqueous systems revisited: II. Alkali metal and halide ion-water interactions. J. Chem. Theory Comput. 2021, 17, 2200-2216. doi:10.1021/acs.jctc.0c01309.

77. Tissandier, M.D.; Cowen, K.A.; Feng, W.Y.; Gundlach, E.; Cohen, M.H.; Earhart, A.D.; Coe, J.V.; Tuttle, Jr., T.R. The proton's absolute aqueous enthalpy and Gibbs free energy of solvation from cluster-ion solvation data. J. Phys. Chem. A 1998, 102, 7787-7794. Erratum: ibid. 102, 9308 (1998), doi:10.1021/jp983807a.

78. Prasetyo, N.; Hünenberger, P.H.; Hofer, T.S. Single-ion thermodynamics from first principles: Calculation of the absolute hydration free energy and single-electrode potential of aqueous $\mathrm{Li}^{+}$using ab initio quantum mechanical/molecular mechanical dynamics simulations. J. Chem. Theory Comput. 2018, 14, 6443-6459. doi:10.1021/acs.jctc.8b00729.

79. Malloum, A.; Fifen, J.J.; Conradie, J. Determination of the absolute solvation free energy and enthalpy of the proton in solutions. J. Mol. Liq. 2021, 322, 114919:1-16. doi:10.1016/j.molliq.2020.114919.

80. Herbert, J.M. Dielectric continuum methods for quantum chemistry. WIREs Comput. Mol. Sci. 2021, 11, e1519:1-73. doi:10.1002/wcms.1519. 
81. Rackers, J.A.; Wang, Q.; Liu, C.; Piquemal, J.P.; Ren, P.; Ponder, J.W. An optimized charge penetration model for use with the AMOEBA force field. Phys. Chem. Chem. Phys. 2017, 19, 276-291. doi:10.1039/C6CP06017J.

82. Deng, S.; Wang, Q.; Ren, P. Estimating and modeling charge transfer from the SAPT induction energy. J. Comput. Chem. 2017, 38, 2222-2231. doi:10.1002/jcc.24864.

83. Jing, Z.; Liu, C.; Ren, P. Advanced electrostatic model for monovalent ions based on ab initio energy decompoosition. J. Chem. Inf. Model 2021, 61, 2806-2817. doi:10.1021/acs.jcim.1c00426.

84. Elgengehi, S.M.; El-Taher, S.; Ibrahim, M.A.A.; El-Kelany, K.E. Unexpected favourable noncovalent interaction between chlorine oxyanions $\left(\mathrm{ClO}_{x}^{-} ; x=1-4\right)$ and benzene: Benchmarking DFT and SAPT methods with respect to CCSD(T). Comput. Theor. Chem. 2021, 1199, 113214:1-10. doi:10.1016/j.comptc.2021.113214.

85. Eklund, L.; Hofer, T.S.; Persson, I. Structure and water exchange dynamics of hydrated oxo halo ions in aqueous solution using QMCF MD simulations, large angle x-ray scattering and EXAFS. Dalton Trans. 2015, 44, 1816-1828. doi:10.1039/C4DT02580F.

86. Eklund, L.; Hofer, T.S.; Pribil, A.B.; Rode, B.M.; Persson, I. On the structure and dynamics of the hydrated sulfite ion in aqueous solution—an ab initio QMCF MD simulation and large angle x-ray scattering study. Dalton Trans. 2012, 41, 5209-5216. doi:10.1039/C2DT12467J.

87. Lao, K.U.; Herbert, J.M. A simple correction for nonadditive dispersion within extended symmetry-adapted perturbation theory (XSAPT). J. Chem. Theory Comput. 2018, 14, 5128-5142. doi:10.1021/acs.jctc.8b00527.

88. Dobson, J.F. Beyond pairwise additivity in London dispersion interactions. Int. J. Quantum Chem. 2014, 114, 1157-1161. doi:10.1002/qua.24635.

89. Tong, Y.; Zhang, I.Y.; Campen, R.K. Experimentally quantifying anion polarizability at the air/water interface. Nat. Commun. 2018, 9, 1313:1-8. doi:10.1038/s41467-018-03598-x.

90. Zhang, Y.; Cremer, P.S. Interactions between macromolecules and ions: The Hofmeister series. Curr. Opin. Struc. Biol. 2006, 10, 659-663. doi:10.1016/j.cbpa.2006.09.020.

91. Jungwirth, P.; Cremer, P.S. Beyond Hofmeister. Nat. Chem. 2014, 6, 261-263. doi:10.1038/nchem.1899.

92. Rembert, K.B.; Paterová, J.; Heyda, J.; Hilty, C.; Jungwirth, P.; Cremer, P.S. Molecular mechanisms of ion-specific effects on proteins. J. Am. Chem. Soc. 2012, 134, 10039-10046. doi:10.1021/ja301297g. 